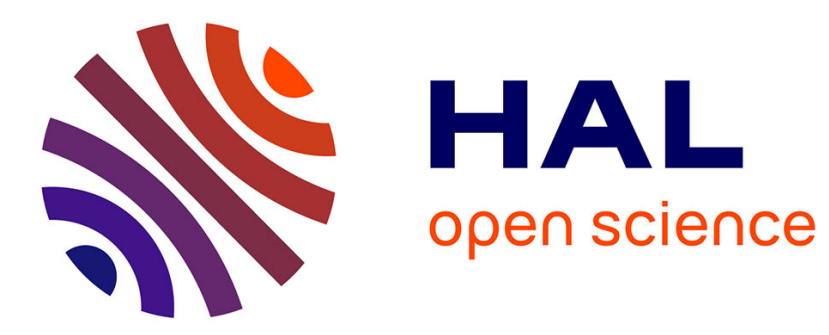

\title{
Generation of accelerograms compatible with design specifications using information theory
}

\author{
Anas Batou, Christian Soize
}

\section{To cite this version:}

Anas Batou, Christian Soize. Generation of accelerograms compatible with design specifications using information theory. Bulletin of Earthquake Engineering, 2014, 12 (2), pp.769-794. 10.1007/s10518013-9547-z . hal-00903236

\section{HAL Id: hal-00903236 \\ https://hal.science/hal-00903236}

Submitted on 10 Nov 2013

HAL is a multi-disciplinary open access archive for the deposit and dissemination of scientific research documents, whether they are published or not. The documents may come from teaching and research institutions in France or abroad, or from public or private research centers.
L'archive ouverte pluridisciplinaire HAL, est destinée au dépôt et à la diffusion de documents scientifiques de niveau recherche, publiés ou non, émanant des établissements d'enseignement et de recherche français ou étrangers, des laboratoires publics ou privés. 


\title{
Generation of accelerograms compatible with design specifications using information theory
}

\author{
Anas Batou · Christian Soize
}

Received: date / Accepted: date

\begin{abstract}
This paper deals with the generation of seismic accelerograms which are compatible with a given response spectrum and other design specifications. The time sampling of the stochastic accelerogram yields a time series represented by a random vector in high dimension. The probability density function of this random vector is constructed using the Maximum Entropy (MaxEnt) principle under constraints defined by the available information (design specifications). In this paper, an adapted algorithm is proposed to identify the Lagrange multipliers introduced in the MaxEnt principle to take into account the constraints. This algorithm is based on (1) the minimization of an appropriate convex functional and (2) the construction of the probability distribution defined as the invariant measure of an Itô Stochastic Differential Equation in order to estimate the integrals in high dimension of the problem. The constraints related to a seismic accelerogram are developed explicitly. This methodology is validated through an application for which the available information is related to the variance of each component of the random vector representing the accelerogram, statistics on the response spectrum, on the Peak Ground Acceleration, on the Cumulative Absolute Velocity and on the end-values for the velocity and for the displacement.
\end{abstract}

Keywords accelerogram - generation of accelerograms · response spectrum · Eurocode $\cdot$ PGA $\cdot$ information theory $\cdot$ maximum entropy principle

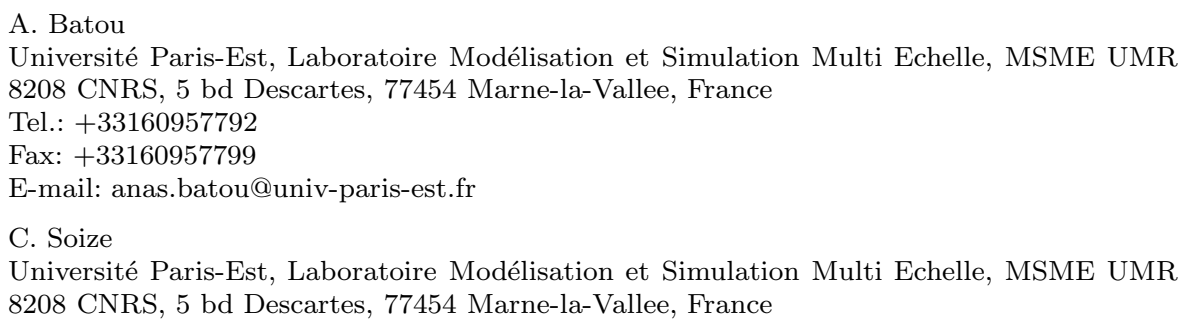




\section{Introduction}

In the context of a seismic risk assessment, the calculation of the transient response of a dynamical structure requires the specification of seismic loads corresponding to specific site characteristics and specific scenarios. These seismic loads can be constructed directly using recorded accelerograms (Katsanos et al (2010); Mukherjee and Gupta (2002); Naeim et al (2004)). This method has the advantage to keep all the features of a natural accelerogram and has to be used whenever a sufficient number of seismic records is available for the site where the structure is located and for the scenario considered by the engineers. Several methods for the selection and the modification of recorded accelerograms have been proposed in the literature in order to extend the applicability domain of the available recorded accelerograms of the world databases (Hancock et al (2006); Mukherjee and Gupta (2002); Naeim et al (2004); Gu and Wen (2007)). In Zentner and Poirion (2012), the authors use a Karhunen-Loève representation to extend a database. This method allows to generate new accelerograms which have the same natural features as the accelerograms from which the Karhunen-Loève representation has been constructed. If no recorded accelerogram corresponding to the studied case is available, then synthetic accelerograms have to be generated in order to perform the seismic transient analysis of the structure. Due to the randomness of seismic signals, these synthetic accelerograms are simulated as realizations of stochastic processes. A large amount of simulation methods have been proposed in the literature. Most of these methods concern the simulation of trajectories of stationary (see for instance Ahmadi (1979); Iyengar and Rao (1979); Preumont (1980); Shrinkhande and Gupta (1996)) or non-stationary (in amplitude Spanos and Loli (1985); Giaralis and Spanos (2012) and in frequency Preumont (1985); Sabetta and Pugliese (1996); Razaeian and Der Kiureghian (2008); Cacciola (2010); Cacciola and Zentner (2012)) Gaussian stochastic processes which are compatible with a response spectrum. Indeed, most of the regulation codes such as the Eurocode 8 (CEN (2003)) only imposes compatibility of the mean value of the velocity response spectrum with the response spectrum which depends on the peak ground acceleration, soil conditions, etc. In Lin and Ghaboussi (2001), the authors introduce stochastic neural networks (for which learning is done using recorded accelerograms) to simulate spectrum-compatible accelerograms. In Giaralis and Spanos (2009), the authors propose a wavelet-based method in order to improve the matching with the target design spectrum. The large quantity of methods proposed in the literature is mainly due to the fact that the solution of the problem consisting in the construction of a centered stochastic process for which the mean velocity response spectrum is equal to a target value is not unique. The Gaussian assumption is often used for controlling the frequency content of the accelerograms but is not imposed by the regulation codes. The Maximum Entropy (MaxEnt) principle (Shannon (1948); Jaynes (1954); Kapur and Kevasan (1992)) is a powerful method which allows a probability distribution of a random vector to be constructed under some constraints defined by the available information. 
A great advantage of this method is that, if it exists, the solution is unique. This method has recently been applied in Soize (2010) for the generation of spectrum-compatible accelerograms as trajectories of a non-stationary nonGaussian centered stochastic process represented by a high-dimension random vector for which the probability density function (pdf) is constructed using the MaxEnt principle under constraints related to (1) the mean value, (2) the variance of the components and (3) the mean value of the velocity response spectrum. The work presented in Soize (2010) was developed in the general context of the generation of any accelerograms compatible with a given velocity response spectrum applied to an equipment or a secondary subsystem and was not specific to a seismic accelerogram.

The methodologies referenced above are mainly focussed on the response spectrum compatibility (which is the main constraint in most of the regulation codes). The objective of this paper is to take into account additional constraints related to (1) some features of natural seismic accelerograms and (2) some accelerogram characteristics used in the earthquake engineering community in order to describe the strong ground motion. To achieve this objective, the methodology proposed in Soize (2010) is extended to take into account, if needed, constraints related to (1) the end-values for the velocity and the displacement, (2) the Peak Ground Acceleration (PGA), (3) the Peak Ground Velocity (PGV) (4) the envelop of the random Velocity Response Spectrum (VRS), (5) the Cumulative Absolute Velocity (CAV) and (6) the Arias Intensity (AI). The probability density function is constructed using the MaxEnt principle and a generator of independent realization adapted to the high stochastic dimension of an accelerogram is proposed. Furthermore an adapted method for the identification of the Lagrange multipliers is proposed in this paper. It should be noted that the methodology proposed for the generation of accelerograms is very versatile and can be adapted, if necessary, for any specific situation of seismic risk assessment.

In Section 2, the MaxEnt principle is used to construct the pdf of the acceleration random vector under constraints defined by general available information. In Section 3, the available information specific to a seismic accelerogram is constructed. Finally, Section 4 is devoted to the application of the methodology for several cases.

\section{Construction of the probability distribution of the sampled seismic accelerograms}

The MaxEnt principle is used to construct the pdf of the random vector corresponding to the time sampling of the stochastic process (modeling the sampled seismic accelerograms) under the constraints defined by the available information. In this section, we summarize the general methodology for the construction of the pdf of such a random vector. The application of the methodology to specific constraints associated with seismic accelerograms will be presented in Section 3. The random acceleration of the soil is modeled by a non-Gaussian 
second-order centered stochastic process $\{A(t), t \in[0, T]\}$. A time sampling with $t_{j}=j \Delta t, j=1, \ldots, N$ and $T=N \times \Delta t$, of this stochastic process is introduced yielding a time series $\left\{A_{1}, \ldots, A_{N}\right\}$ with $A_{j}=A\left(t_{j}\right)$ and for which the random vector $\boldsymbol{A}=\left(A_{1}, \ldots, A_{N}\right)$ is associated with. Finally, we have to construct the probability distribution of random vector $\boldsymbol{A}$.

\subsection{Maximum entropy principle}

In this paper, the upper case boldface letter $\boldsymbol{A}$ corresponds to a random vector. The probability density function of random vector $\boldsymbol{A}$ is denoted by $p_{\boldsymbol{A}}(\boldsymbol{a})$ (or by $p_{\boldsymbol{A}}\left(a_{1}, \ldots, a_{N}\right)$ and is such that the probability of the event, $\left\{A_{1} \leq\right.$ $\left.a_{1}^{*}, \ldots, A_{N} \leq a_{N}^{*}\right\}$, is written (cumulative distribution function) as

$$
\operatorname{Proba}\left\{A_{1} \leq a_{1}^{*}, \ldots, A_{N} \leq a_{N}^{*}\right\}=\int_{-\infty}^{a_{1}^{*}} \ldots \int_{-\infty}^{a_{N}^{*}} p_{\boldsymbol{A}}\left(a_{1}, \ldots, a_{N}\right) d a_{1} \ldots d a_{N},
$$

in which the volume element $d a_{1} \ldots d a_{N}$ is also denoted by $d \boldsymbol{a}$. The lower case boldface letter $\boldsymbol{a}=\left(a_{1}, \ldots, a_{N}\right)$ will denote the deterministic vector (with deterministic components $a_{1}, \ldots, a_{N}$ ) associated with random vector $\boldsymbol{A}=\left(A_{1}, \ldots, A_{N}\right)$ (with random components $A_{1}, \ldots, A_{N}$ ) and will correspond to an integration variable associated with the random vector $\boldsymbol{A}$ or will correspond to any deterministic value associated with $\boldsymbol{A}$. For instance, if $E\{g(\boldsymbol{A})\}$ denotes the mathematical expectation of the random variable $g(\boldsymbol{A})$ in which $g$ is a deterministic real function, this function will be denoted as $g(\boldsymbol{a})$. It can also be said that the deterministic vector $\boldsymbol{a}=\left(a_{1}, \ldots, a_{N}\right)$ represents any possible value of random vector $\boldsymbol{A}=\left(A_{1}, \ldots, A_{N}\right)$ that is to say, any possible value of the time sampling $\boldsymbol{A}=\left(A\left(t_{1}\right), \ldots, A\left(t_{N}\right)\right)$ of the stochastic process $\{A(t), t \in[0, T]\}$ modeling the soil acceleration.

The objective of this section is to construct the $\operatorname{pdf} p_{\boldsymbol{A}}(\boldsymbol{a})$ of random vector $\boldsymbol{A}$. This construction is performed by using the MaxEnt principle under the constraints defined by the available information related to random vector $\boldsymbol{A}$. The available information is written as

$$
E\{\boldsymbol{g}(\boldsymbol{A})\}=\boldsymbol{f},
$$

in which $\boldsymbol{g}(\boldsymbol{a})=\left(g_{1}(\boldsymbol{a}), \ldots, g_{\mu}(\boldsymbol{a})\right)$ is a given function which is defined herein as the information function and where $\boldsymbol{f}=\left(f_{1}, \ldots, f_{\mu}\right)$ is a given vector (or target). Equation (1) can be rewritten as

$$
\int_{\mathbb{R}^{N}} \boldsymbol{g}(\boldsymbol{a}) p_{\boldsymbol{A}}(\boldsymbol{a}) \mathrm{d} \boldsymbol{a}=\boldsymbol{f} .
$$

An additional constraint related to the normalization of the pdf $p_{\boldsymbol{A}}$ is introduced such that

$$
\int_{\mathbb{R}^{N}} p_{\boldsymbol{A}}(\boldsymbol{a}) \mathrm{d} \boldsymbol{a}=1
$$


The entropy of pdf $p_{\boldsymbol{A}}$ is defined by

$$
S\left(p_{\boldsymbol{A}}\right)=-\int_{\mathbb{R}^{N}} p_{\boldsymbol{A}}(\boldsymbol{a}) \log \left(p_{\boldsymbol{A}}(\boldsymbol{a})\right) \mathrm{d} \boldsymbol{a},
$$

where $\log$ is the natural logarithm. This functional measures the related uncertainty of $p_{\boldsymbol{A}}$. Let $\mathcal{C}$ be the set of all the possible pdfs of random vector $\boldsymbol{A}$, satisfying the constraints defined by Eqs. (2) and (3). Then the MaxEnt principle consists in constructing the pdf $p_{\boldsymbol{A}}$ as the unique pdf in $\mathcal{C}$ which maximizes entropy $S\left(p_{\boldsymbol{A}}\right)$. Then by introducing a positive Lagrange multiplier $\lambda_{0}$ associated with Eq. (3) and a Lagrange multiplier $\boldsymbol{\lambda}=\left(\lambda_{1}, \ldots, \lambda_{\mu}\right)$ associated with Eq. (2) and belonging to an admissible set $\mathcal{L}_{\mu}$, it can be shown (see Jaynes (1954); Kapur and Kevasan (1992)) that the MaxEnt solution, if it exists, is defined by

$$
p_{\boldsymbol{A}}(\boldsymbol{a})=c_{0}^{\mathrm{sol}} \exp \left(-\left\langle\boldsymbol{\lambda}^{\mathrm{sol}}, \boldsymbol{g}(\boldsymbol{a})\right\rangle\right)
$$

in which $c_{0}^{\text {sol }}=\exp \left(-\lambda_{0}^{\text {sol }}\right),\langle\boldsymbol{x}, \boldsymbol{y}\rangle=x_{1} y_{1}+\ldots+x_{\mu} y_{\mu}$ and where $\lambda_{0}^{\text {sol }}$ and $\boldsymbol{\lambda}^{\text {sol }}$ are respectively the values of $\lambda_{0}$ and $\boldsymbol{\lambda}$ for which Eqs. (2) and (3) are satisfied. Using Eqs. (3) and (5), the parameter $c_{0}^{\text {sol }}$ can be rewritten as a function of $\lambda^{\text {sol }}$ and Eq. (6) can be rewritten as

$$
p_{\boldsymbol{A}}(\boldsymbol{a})=c_{0}\left(\boldsymbol{\lambda}^{\mathrm{sol}}\right) \exp \left(-\left\langle\boldsymbol{\lambda}^{\mathrm{sol}}, \boldsymbol{g}(\boldsymbol{a})\right\rangle\right),
$$

in which $\boldsymbol{\lambda}^{\text {sol }}$ is such that Eq. (2) is satisfied and where $c_{0}(\boldsymbol{\lambda})$ is defined by

$$
c_{0}(\boldsymbol{\lambda})=\left\{\int_{\mathbb{R}^{N}} \exp (-\langle\boldsymbol{\lambda}, \boldsymbol{g}(\boldsymbol{a})\rangle) \mathrm{d} \boldsymbol{a}\right\}^{-1} .
$$

At this stage, a parametric MaxEnt pdf $p_{\boldsymbol{A}}$ has been constructed but the vector of the Lagrange multipliers $\boldsymbol{\lambda}^{\text {sol }}$ has not been calculated yet. This is the objective of the next section.

\subsection{Calculation of the Lagrange multipliers}

In this section, we propose a methodology for the calculation of $\boldsymbol{\lambda}^{\text {sol }}$. We first present the general methodology for the calculation of this vector. Then the Gaussian particular case is presented for the case for which the gradient and the Hessian of the objective function can explicitly be calculated. Note that the Gaussian case will be useful (1) either if the available information consists only of linear or affine transformations of statistical second-order moments (2) or, for initializing the value of the Lagrange multipliers in the iterative algorithm which will be presented for the non-Gaussian case. Finally, for the general non-Gaussian case, we will present a generator of independent realizations of random vector $\boldsymbol{A}$, which is adapted to the high dimension. 
2.2.1 Objective function and methododology

Using Eqs. (6) and (2), vector $\boldsymbol{\lambda}^{\text {sol }}$ is the solution in $\boldsymbol{\lambda}$ of the following nonlinear algebraic equation,

$$
\int_{\mathbb{R}^{N}} \boldsymbol{g}(\boldsymbol{a}) c_{0}(\boldsymbol{\lambda}) \exp (-\langle\boldsymbol{\lambda}, \boldsymbol{g}(\boldsymbol{a})\rangle)=\boldsymbol{f} .
$$

Instead of directly solving Eq. (8), a more convenient method to calculate vector $\boldsymbol{\lambda}^{\text {sol }}$ consists in solving the following optimization problem (see Agmon et al (1979); Golan et al (1996)),

$$
\boldsymbol{\lambda}^{\mathrm{sol}}=\arg \min _{\boldsymbol{\lambda} \in \mathcal{L}_{\mu} \subset \mathbb{R}^{\mu}} \Gamma(\boldsymbol{\lambda}),
$$

in which the objective function $\Gamma$ is written as

$$
\Gamma(\boldsymbol{\lambda})=\langle\boldsymbol{\lambda}, \boldsymbol{f}\rangle-\log \left(c_{0}(\boldsymbol{\lambda})\right) .
$$

It should be noted that the great advantage of such a formulation is that the introduced objective function $\Gamma$ does not depend on the Lagrange multiplier $\lambda_{0}$ associated with the constant of normalization. Let $\left\{\boldsymbol{A}_{\boldsymbol{\lambda}}, \boldsymbol{\lambda} \in \mathcal{L}_{\mu}\right\}$ be a family of random variables for which the pdf is defined, for all $\boldsymbol{\lambda}$ in $\mathcal{L}_{\mu}$, by

$$
p_{\boldsymbol{A}_{\boldsymbol{\lambda}}}(\boldsymbol{a})=c_{0}(\boldsymbol{\lambda}) \exp (-\langle\boldsymbol{\lambda}, \boldsymbol{g}(\boldsymbol{a})\rangle) .
$$

We then have, $\boldsymbol{A}=\boldsymbol{A}_{\boldsymbol{\lambda}^{\text {sol }}}$. The gradient vector $\boldsymbol{\nabla} \Gamma(\boldsymbol{\lambda})$ and the Hessian matrix $[H(\boldsymbol{\lambda})]$ of function $\Gamma(\boldsymbol{\lambda})$ are written as

$$
\begin{gathered}
\boldsymbol{\nabla} \Gamma(\boldsymbol{\lambda})=\boldsymbol{f}-E\left\{\boldsymbol{g}\left(\boldsymbol{A}_{\boldsymbol{\lambda}}\right)\right\}, \\
{[H(\boldsymbol{\lambda})]=E\left\{\boldsymbol{g}\left(\boldsymbol{A}_{\boldsymbol{\lambda}}\right) \boldsymbol{g}\left(\boldsymbol{A}_{\boldsymbol{\lambda}}\right)^{T}\right\}-E\left\{\boldsymbol{g}\left(\boldsymbol{A}_{\boldsymbol{\lambda}}\right)\right\} E\left\{\boldsymbol{g}\left(\boldsymbol{A}_{\boldsymbol{\lambda}}\right)\right\}^{T},}
\end{gathered}
$$

in which $\boldsymbol{u}^{T}$ is the transpose of $\boldsymbol{u}$. It can be noted that the Hessian matrix $[H(\boldsymbol{\lambda})]$ is the covariance matrix of the random vector $\boldsymbol{g}\left(\boldsymbol{A}_{\boldsymbol{\lambda}}\right)$. It is assumed that the constraints defined by Eq. (2) are algebraically independent. Consequently, the Hessian matrix is positive definite and therefore, function $\Gamma$ is strictly convex and reaches its minimum for $\boldsymbol{\lambda}^{\text {sol }}$ which is such that $\boldsymbol{\nabla} \Gamma(\boldsymbol{\lambda})=\mathbf{0}$ for $\boldsymbol{\lambda}=\boldsymbol{\lambda}^{\mathrm{sol}}$. It can then be deduced that the minimum of function $\Gamma$ corresponds to the solution of Eq. (8). The optimization problem defined by Eq. (9) can be solved using any minimization algorithm. The Newton iterative method can be applied to the increasing function $\boldsymbol{\nabla} \Gamma$ for searching $\boldsymbol{\lambda}^{\mathrm{sol}}$ such that $\boldsymbol{\nabla} \Gamma\left(\boldsymbol{\lambda}^{\mathrm{sol}}\right)=$ 0. This iterative method is not unconditionally convergent. Consequently, an under-relaxation is introduced and the iterative algorithm is written as

$$
\boldsymbol{\lambda}^{\mathrm{i}+1}=\boldsymbol{\lambda}^{\mathrm{i}}-\alpha\left[H\left(\boldsymbol{\lambda}^{\mathrm{i}}\right)\right]^{-1} \nabla \Gamma\left(\boldsymbol{\lambda}^{\mathrm{i}}\right),
$$

in which $\alpha$ belongs to $] 0,1]$ in order to ensure the convergence towards the solution $\lambda^{\text {sol }}$. 


\subsubsection{Gaussian case}

In this section, it is assumed that the available information leads us to a Gaussian second-order centered random vector $\boldsymbol{A}$. This particular case is interesting for calculating the covariance matrix of the Gaussian centered random vector $\boldsymbol{A}$ when the $\mu$ constraints are defined by $\mu$ linear (or affine) functions of the second-order moments. In the case of a seismic accelerogram, this type of information is useful to define the time envelop of the stochastic process and also to control the velocity and displacement traces. Since these constraints must be algebraically independent, it is assumed that $1<\mu \leq N(N+1) / 2$. The available information is then defined as follows,

$$
\begin{gathered}
E\{\boldsymbol{A}\}=\mathbf{0}, \\
E\left\{\frac{1}{2}\left\langle\boldsymbol{A},\left[K_{j}\right] \boldsymbol{A}\right\rangle\right\}=h_{j} \quad, \quad j=1, \ldots, \mu,
\end{gathered}
$$

in which $\left[K_{1}\right], \ldots,\left[K_{\mu}\right]$ are $\mu$ symmetric $(N \times N)$ real matrices which are assumed to be algebraically independent. In this case, it can be shown that the Lagrange multipliers vector related to the constraint defined by Eq. (15) is zero and therefore the MaxEnt pdf is written as

$$
p_{\boldsymbol{A}_{\boldsymbol{\lambda}}}(\boldsymbol{a} ; \boldsymbol{\lambda})=c_{0}(\boldsymbol{\lambda}) \exp \left(-\frac{1}{2}\left\langle\boldsymbol{a},\left[\mathcal{K}_{\boldsymbol{\lambda}}\right] \boldsymbol{a}\right\rangle\right),
$$

in which $\left[\mathcal{K}_{\boldsymbol{\lambda}}\right]=\sum_{j=1}^{\mu} \lambda_{j}\left[K_{j}\right]$ is assumed to be positive definite for all $\boldsymbol{\lambda}$ in $\mathcal{L}_{\mu}$ and where $c_{0}(\boldsymbol{\lambda})$ is the normalization constant such that

$$
c_{0}(\boldsymbol{\lambda})=(2 \pi)^{-\frac{N}{2}} \sqrt{\operatorname{det}\left[\mathcal{K}_{\boldsymbol{\lambda}}\right]} .
$$

Equations (17) and (18) show that $\boldsymbol{A}_{\boldsymbol{\lambda}}$ is a real centered Gaussian random vector for which the covariance matrix is $[\mathcal{K}(\boldsymbol{\lambda})]^{-1}$. For this Gaussian case, $\Gamma(\boldsymbol{\lambda}), \nabla \Gamma(\boldsymbol{\lambda})$ and $[H(\boldsymbol{\lambda})]$ can explicitly be derived. The use of Eqs. (10) and (18) yields

$$
\Gamma(\boldsymbol{\lambda})=\langle\boldsymbol{\lambda}, \boldsymbol{h}\rangle+\frac{N}{2} \log (2 \pi)-\frac{1}{2} \log \left(\operatorname{det}\left(\left[\mathcal{K}_{\boldsymbol{\lambda}}\right]\right) .\right.
$$

in which $\boldsymbol{h}=\left(h_{1}, \ldots, h_{\mu}\right)$. The gradient vector is then written as

$$
(\nabla \Gamma(\boldsymbol{\lambda}))_{i}=h_{i}-\frac{1}{2} \operatorname{tr}\left(\left[\mathcal{K}_{\boldsymbol{\lambda}}\right]^{-1}\left[K_{i}\right]\right)
$$

in which $\operatorname{tr}$ is the trace for matrices. The Hessian matrix is written as

$$
[H(\boldsymbol{\lambda})]_{i j}=\frac{1}{2} \operatorname{tr}\left(\left[\mathcal{K}_{\boldsymbol{\lambda}}\right]^{-1}\left[K_{i}\right]\left[\mathcal{K}_{\boldsymbol{\lambda}}\right]^{-1}\left[K_{j}\right]\right) .
$$

Finally, Eq. (14) allows $\boldsymbol{\lambda}^{\text {sol }}$ to be calculated iteratively. 


\subsubsection{General non-Gaussian case}

In general, for the non-Gaussian case, the $N$-dimensional integrals appearing in Eqs. (12) and (13) cannot explicitly (such as in the Gaussian case) be calculated and cannot be discretized for large value of $N$. In this paper, these integrals are estimated using the Monte Carlo simulation method (Rubinstein and Kroese (2008)) for which the convergence rate is independent of $N$ and for which independent realizations of random vector $\boldsymbol{A}_{\boldsymbol{\lambda}}$ are generated using an algorithm belonging to the Markov Chain Monte Carlo (MCMC) which is adapted to the high stochastic dimension, as proposed in Soize $(2008,2010)$. In this Section, we first introduce a generator of independent realizations adapted to random vectors in high-dimension for which the pdf is constructed using the MaxEnt principle. Then, the integrals in Eqs. (12) and (13) are estimated. Finally, an algorithm for the calculation of vector $\lambda^{\text {sol }}$ of the Lagrange multipliers is presented.

\section{$i$ - Generator of independent realizations}

The objective of this section is to provide a generator of independent realizations of the random variable $\boldsymbol{A}_{\boldsymbol{\lambda}}$ for all $\boldsymbol{\lambda}$ fixed in $\mathcal{L}_{\mu}$. A generator of independent realizations for MaxEnt distributions has been proposed in Soize $(2008,2010)$ in the class of the MCMC algorithms. This methodology consists in constructing the pdf of random vector $\boldsymbol{A}_{\boldsymbol{\lambda}}$ as the pdf $p_{\boldsymbol{A}_{\boldsymbol{\lambda}}}$ associated with the stationary solution of a second-order nonlinear Itô Stochastic Differential Equation (ISDE). The advantages of this generator compared to the other MCMC generators such as the Metropolis-Hastings algorithm (see Hastings (1970)) are: (1) The mathematical results concerning the existence and the uniqueness of an invariant measure can be used, (2) a damping matrix can be introduced in order to rapidly reach the invariant measure and (3) there is no need to introduce a proposal distribution which can induce difficulties in high dimension. Below, we directly introduce the generator of independent realizations using a discretization of the ISDE. Details concerning the construction of this generator can be found in Soize $(2008,2010)$.

As proposed in Soize $(2008,2010)$, the ISDE is discretized using a semiimplicit integration scheme in order to avoid the resolution of an algebraic nonlinear equation at each step while allowing significantly increase of the time step compared to a purely explicit scheme. We assume that function $\boldsymbol{g}$, introduced in Eq. (1) and defining the available information, is written as $\boldsymbol{g}(\boldsymbol{a})=\left(\boldsymbol{g}_{\mathrm{L}}(\boldsymbol{a}), \boldsymbol{g}_{\mathrm{NL}}(\boldsymbol{a})\right)$ in which $\boldsymbol{g}_{\mathrm{NL}}=\left(\left\{\boldsymbol{g}_{\mathrm{NL}}\right\}_{1}, \ldots,\left\{\boldsymbol{g}_{\mathrm{NL}}\right\}_{\mu_{\mathrm{NL}}}\right)$ is a nonlinear function and where $\boldsymbol{g}_{\mathrm{L}}=\left(\left\{\boldsymbol{g}_{\mathrm{L}}\right\}_{1}, \ldots,\left\{\boldsymbol{g}_{\mathrm{L}}\right\}_{\mu_{\mathrm{L}}}\right)$ is a quadratic function whose components are such that

$$
\left\{\boldsymbol{g}_{\mathrm{L}}(\boldsymbol{a})\right\}_{i}=\frac{1}{2}\left\langle\boldsymbol{a},\left[K_{i}\right] \boldsymbol{a}\right\rangle,
$$


in which $\left[K_{1}\right], \ldots,\left[K_{\mu_{\mathrm{L}}}\right]$ are $\mu_{\mathrm{L}}$ symmetric $(N \times N)$ real matrices which are assumed to be algebraically independent. Let $\Phi(\boldsymbol{a}, \boldsymbol{\lambda})$ be a potential function in $\boldsymbol{a}$ defined by

$$
\Phi(\boldsymbol{a}, \boldsymbol{\lambda})=\langle\boldsymbol{\lambda}, \boldsymbol{g}(\boldsymbol{a})\rangle
$$

Let us introduce the decomposition $\boldsymbol{\lambda}=\left(\boldsymbol{\lambda}_{\mathrm{L}}, \boldsymbol{\lambda}_{\mathrm{NL}}\right) \in \mathcal{L}_{\mu}=\mathcal{L}_{\mu_{\mathrm{L}}} \times \mathcal{L}_{\mu_{\mathrm{NL}}}$ of the Lagrange multipliers. Then for all $\boldsymbol{\lambda} \in \mathcal{L}_{\mu}$, the potential function can be written as

$$
\Phi(\boldsymbol{a}, \boldsymbol{\lambda})=\frac{1}{2}\left\langle\boldsymbol{a},\left[\mathcal{K}_{\boldsymbol{\lambda}_{\mathrm{L}}}\right] \boldsymbol{a}\right\rangle+\Phi_{\mathrm{NL}}\left(\boldsymbol{a}, \boldsymbol{\lambda}_{\mathrm{NL}}\right),
$$

in which the symmetric matrix $\left[\mathcal{K}_{\boldsymbol{\lambda}_{\mathrm{L}}}\right]=\sum_{j=1}^{\mu_{\mathrm{L}}}\left\{\boldsymbol{\lambda}_{\mathrm{L}}\right\}_{j}\left[K_{j}\right]$ is assumed to be positive definite for all $\boldsymbol{\lambda}_{\mathrm{L}}$ in $\mathcal{L}_{\mu_{\mathrm{L}}}$ and where $\Phi_{\mathrm{NL}}\left(\boldsymbol{a}, \boldsymbol{\lambda}_{\mathrm{NL}}\right)=\sum_{j=1}^{\mu_{\mathrm{NL}}}\left\{\boldsymbol{\lambda}_{\mathrm{NL}}\right\}_{j}\left\{\boldsymbol{g}_{\mathrm{NL}}(\boldsymbol{a})\right\}_{j}$. Therefore, the gradient of the potential function with respect to $\boldsymbol{a}$ is written as

$$
\nabla_{\boldsymbol{a}} \Phi(\boldsymbol{a}, \boldsymbol{\lambda})=\left[\mathcal{K}_{\boldsymbol{\lambda}_{\mathrm{L}}}\right] \boldsymbol{a}+\boldsymbol{\nabla}_{\boldsymbol{a}} \Phi_{\mathrm{NL}}\left(\boldsymbol{a}, \boldsymbol{\lambda}_{\mathrm{NL}}\right)
$$

in which $\boldsymbol{\nabla}_{\boldsymbol{a}} \Phi_{\mathrm{NL}}\left(\boldsymbol{a}, \boldsymbol{\lambda}_{\mathrm{NL}}\right)=\sum_{j=1}^{\mu_{\mathrm{NL}}}\left\{\boldsymbol{\lambda}_{\mathrm{NL}}\right\}_{j} \boldsymbol{\nabla}_{\boldsymbol{a}}\left\{\boldsymbol{g}_{\mathrm{NL}}(\boldsymbol{a})\right\}_{j}$. Thus the gradient function $\nabla_{\boldsymbol{a}} \Phi(\boldsymbol{a}, \boldsymbol{\lambda})$ is decomposed into a linear part with respect to $\boldsymbol{a}$ and a nonlinear part.

Let $\Delta r_{\boldsymbol{\lambda}}$ be the integration step size related to the discretization of the ISDE. Let $\Delta \boldsymbol{W}^{1}, \ldots, \Delta \boldsymbol{W}^{M}$ be $M$ mutually independent second-order Gaussian centered random vector with covariance matrix equal to $\Delta r_{\boldsymbol{\lambda}}\left[I_{N}\right]$. We introduce the time series $\left\{\left(\boldsymbol{U}^{k}, \boldsymbol{V}^{k}\right), k=1, \ldots, M\right\}$ with $\boldsymbol{U}^{k}=\left(U_{1}^{k}, \ldots, U_{N}^{k}\right)$ and $\boldsymbol{V}^{k}=\left(V_{1}^{k}, \ldots, V_{N}^{k}\right)$. The corresponding initial conditions $\left(\boldsymbol{U}_{0}, \boldsymbol{V}_{0}\right)$ are assumed to be second-order random vectors which are independent of time series $\left\{\left(\boldsymbol{U}^{k}, \boldsymbol{V}^{k}\right), k=1, \ldots, M\right\}$.

For $\ell=1, \ldots, n_{s}$, using $n_{s}$ independent realizations $\left\{\Delta \boldsymbol{W}^{k+1, \ell}, k=1, \ldots\right.$, $M-1\}$ of the family of random vectors $\left\{\Delta \boldsymbol{W}^{k+1}, k=1, \ldots, M-1\right\}$ and $n_{s}$ independent realizations $\left(\boldsymbol{U}_{0}^{\ell}, \boldsymbol{V}_{0}^{\ell}\right)$ of the random initial conditions $\left(\boldsymbol{U}_{0}, \boldsymbol{V}_{0}\right)$ (which are also independent of $\left\{\Delta \boldsymbol{W}^{k+1}, k=1, \ldots, M-1\right\}$ ), then the $n_{s}$ independent realizations $\boldsymbol{U}^{M, \ell}$ of the vector random $\boldsymbol{U}^{M}$ are generated by solving $n_{s}$ times, for $k=1, \ldots, M-1$, the following equations (which correspond to the discretization of the ISDE, see Soize (2008))

$$
\begin{aligned}
& {\left[\mathcal{E}_{\boldsymbol{\lambda}}\right] \boldsymbol{V}^{k+1, \ell} }=\left[\mathcal{B}_{\boldsymbol{\lambda}}\right] \boldsymbol{V}^{k, \ell}-\Delta r_{\boldsymbol{\lambda}}\left[\mathcal{K}_{\boldsymbol{\lambda}_{\mathrm{L}}}\right] \boldsymbol{U}^{k, \ell}+\Delta r_{\boldsymbol{\lambda}} \boldsymbol{L}_{\mathrm{NL}}^{k, \ell}+\left[S_{\boldsymbol{\lambda}}\right] \Delta \boldsymbol{W}^{k+1, \ell}, \\
& \boldsymbol{U}^{k+1, \ell}=\boldsymbol{U}^{k, \ell}+\frac{1}{2} \Delta r_{\boldsymbol{\lambda}}\left(\boldsymbol{V}^{k+1, \ell}+\boldsymbol{V}^{k, \ell}\right) \\
& \boldsymbol{U}^{1, \ell}=\boldsymbol{U}_{0}^{\ell} \quad, \quad \boldsymbol{V}^{1, \ell}=\boldsymbol{V}_{0}^{\ell} .
\end{aligned}
$$

in which $\left[\mathcal{E}_{\boldsymbol{\lambda}}\right]=\left[I_{N}\right]+\frac{1}{4} \Delta r_{\boldsymbol{\lambda}}\left[D_{\boldsymbol{\lambda}}\right]+\frac{1}{4} \Delta r_{\boldsymbol{\lambda}}^{2}\left[\mathcal{K}_{\boldsymbol{\lambda}_{\mathrm{L}}}\right]$ and $\left[\mathcal{B}_{\boldsymbol{\lambda}}\right]=\left[I_{N}\right]-\frac{1}{4} \Delta r_{\boldsymbol{\lambda}}\left[D_{\boldsymbol{\lambda}}\right]-$ $\frac{1}{4} \Delta r_{\boldsymbol{\lambda}}^{2}\left[\mathcal{K}_{\boldsymbol{\lambda}_{\mathrm{L}}}\right]$ where $\left[I_{N}\right]$ is the $(N \times N)$ identity matrix and where $\left[D_{\boldsymbol{\lambda}}\right]$ is a symmetric positive-definite damping matrix and the lower triangular matrix $\left[S_{\boldsymbol{\lambda}}\right]$ is such that $\left[D_{\boldsymbol{\lambda}}\right]=\left[S_{\boldsymbol{\lambda}}\right]\left[S_{\boldsymbol{\lambda}}\right]^{T}$. The vector $\boldsymbol{L}_{\mathrm{NL}}^{k}$ is defined by $\boldsymbol{L}_{\mathrm{NL}}^{k}=$ 
$-\left\{\boldsymbol{\nabla}_{\boldsymbol{a}} \Phi_{\mathrm{NL}}\left(\boldsymbol{a}, \boldsymbol{\lambda}_{\mathrm{NL}}\right)\right\}_{\boldsymbol{a}=\boldsymbol{U}^{k}}$. If the gradient function $\boldsymbol{\nabla}_{\boldsymbol{a}} \Phi_{\mathrm{NL}}\left(\boldsymbol{a}, \boldsymbol{\lambda}_{\mathrm{NL}}\right)$ cannot explicitly be calculated, then $\boldsymbol{L}_{\mathrm{NL}}^{k}$ is calculated using a finite difference approximation of $\boldsymbol{\nabla}_{\boldsymbol{a}} \Phi_{\mathrm{NL}}\left(\boldsymbol{a}, \boldsymbol{\lambda}_{\mathrm{NL}}\right)$.

Then, if $M$ is sufficiently large, the $n_{s}$ independent realizations of the random vector $\boldsymbol{A}_{\boldsymbol{\lambda}}$ are constructed such that $\boldsymbol{A}_{\boldsymbol{\lambda}}^{\ell} \simeq \boldsymbol{U}^{M, \ell}$ for $\ell=1, \ldots, n_{s}$. Concerning the initial conditions, the more the probability distribution of the initial conditions is close to the invariant measure, the shorter is the transient response. Finally, the choice of the pdf $p_{\boldsymbol{U}_{0}, \boldsymbol{V}_{0}}$ for the random vector of the initial condition $\left(\boldsymbol{U}_{0}, \boldsymbol{V}_{0}\right)$ is discussed later.

\section{ii - Estimation of the mathematical expectations}

The mean value $E\left\{\boldsymbol{g}\left(\boldsymbol{A}_{\boldsymbol{\lambda}}\right)\right\}$ and the correlation matrix $E\left\{\boldsymbol{g}\left(\boldsymbol{A}_{\boldsymbol{\lambda}}\right) \boldsymbol{g}\left(\boldsymbol{A}_{\boldsymbol{\lambda}}\right)^{T}\right\}$ are estimated using the classical statistical estimators,

$$
\begin{aligned}
E\left\{\boldsymbol{g}\left(\boldsymbol{A}_{\boldsymbol{\lambda}}\right)\right\} & \simeq \frac{1}{n_{s}} \sum_{\ell=1}^{n_{s}} \boldsymbol{g}\left(\boldsymbol{A}_{\boldsymbol{\lambda}}^{\ell}\right), \\
E\left\{\boldsymbol{g}\left(\boldsymbol{A}_{\boldsymbol{\lambda}}\right) \boldsymbol{g}\left(\boldsymbol{A}_{\boldsymbol{\lambda}}\right)^{T}\right\} & \simeq \frac{1}{n_{s}} \sum_{\ell=1}^{n_{s}} \boldsymbol{g}\left(\boldsymbol{A}_{\boldsymbol{\lambda}}^{\ell}\right) \boldsymbol{g}\left(\boldsymbol{A}_{\boldsymbol{\lambda}}^{\ell}\right)^{T} .
\end{aligned}
$$

As previously explained, we need not to calculate the normalization constant $c_{0}(\boldsymbol{\lambda})$ with the proposed algorithm.

iii - Implementation of the iterative algorithm for the calculation of the Lagrange multipliers

Concerning the initialization of the algorithm, an initial value $\boldsymbol{\lambda}_{\text {init }}$ of $\boldsymbol{\lambda}$ has to be provided in $\mathcal{L}_{\mu}$. A pdf of the random vector $\left(\boldsymbol{U}_{0 \text {,init }}, \boldsymbol{V}_{0 \text {,init }}\right)$ corresponding to the random initial condition $\left(\boldsymbol{U}_{0}, \boldsymbol{V}_{0}\right)$ for $\boldsymbol{\lambda}=\boldsymbol{\lambda}_{\text {init }}$ has to be provided too. Concerning $\boldsymbol{V}_{0 \text {,init }}$, it can be proven (see Soize (2008)) that for $M$ sufficiently large, $\boldsymbol{V}^{M} \sim \mathcal{N}\left(\mathbf{0},\left[I_{n}\right]\right)$. Therefore the pdf of $\boldsymbol{V}_{0 \text {,init }}$ is chosen as the normalized Gaussian distribution, $\boldsymbol{V}_{0 \text {,init }} \sim \mathcal{N}\left(\mathbf{0},\left[I_{n}\right]\right)$. Concerning $\boldsymbol{U}_{0 \text {,init }}$, if we set $\boldsymbol{\lambda}_{\mathrm{NL} \text {,init }}=\mathbf{0}$, then $\boldsymbol{A}_{\boldsymbol{\lambda}_{\text {init }}}$ is a Gaussian centered random vector for which the covariance matrix is $\left[\mathcal{K}_{\boldsymbol{\lambda}_{\mathrm{L}, \text { init }}}\right]^{-1}$. Therefore the pdf of $\boldsymbol{U}_{0 \text {,init }}$ is chosen such that $\boldsymbol{U}_{0 \text {,init }} \sim \mathcal{N}\left(\mathbf{0},\left[\mathcal{K}_{\boldsymbol{\lambda}_{\mathrm{L}, \text { init }}}\right]^{-1}\right)$, and the initial value $\boldsymbol{\lambda}_{\mathrm{L} \text {,init }}$ can be identified using the methodology developed in Section 2.2.2 devoted to the Gaussian case. Then $\boldsymbol{\lambda}_{\mathrm{NL} \text {,init }}$ must be chosen such that $\left(\boldsymbol{\lambda}_{\mathrm{L}, \text { init }}, \boldsymbol{\lambda}_{\mathrm{NL} \text {,init }}\right)$ is in $\mathcal{L}_{\mu}$.

At each iteration of the algorithm, the calculation of $\boldsymbol{\lambda}^{i+1}$ given $\boldsymbol{\lambda}^{i}$ requires the calculation of the gradient and the Hessian for $\boldsymbol{\lambda}^{i}$. These quantities are estimated solving the ISDE with the pdf of the random initial condition which is chosen as the pdf of the invariant measure constructed for $\lambda^{i-1}$ for which independent realizations are known. The algorithm for the identification of the Lagrange multipliers is summarized in Table 1. 
Table 1 Identification of the Lagrange multipliers.

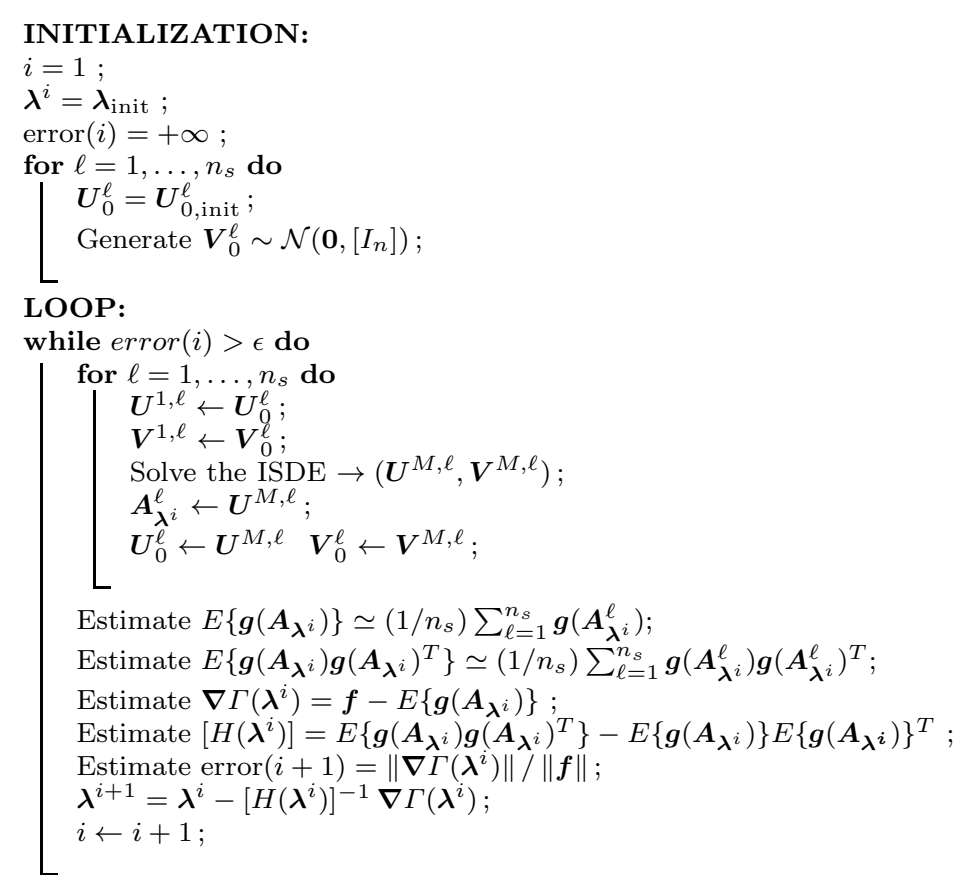

It should be noted that the updating of vector $\boldsymbol{\lambda}$ is sequential but for each value of $\boldsymbol{\lambda}$, the $n_{s}$ Monte Carlo loops can be calculated on parallel processors.

\section{Examples of information related to seismic accelerograms}

In this section, some examples of information related to a seismic accelerogram is presented. This list is not exhaustive and can be completed if necessary. For each part of available information, the information function $\boldsymbol{g}(\boldsymbol{a})$ and its gradient, which are necessary for the algorithm introduced in Section 2.2.3 are specified in Appendix A.

\subsection{Mean value}

The seismic accelerogram is modeled by a centered stochastic process. Therefore the vector $\boldsymbol{A}$ has to be centered. We then have the constraint

$$
E\{\boldsymbol{A}\}=\mathbf{0}
$$


3.2 Variance of the components

This constraint allows the envelop of the accelerogram to be specified and therefore the strong motion duration to be fixed. Since random vector must be centered, it is equivalent to impose the variance of the components or their second-order moments. For $j=\{1, \ldots, N\}$, these constraints are defined by

$$
E\left\{A_{j}^{2}\right\}=\sigma_{j}^{2}<+\infty .
$$

3.3 Variance of the end-velocity and the end-displacement

The objective of this constraint is to control the end-velocity and the enddisplacement which are assumed to be zero. Usually, this control is carried out using an adapted filtering to perform a correction of recorded or simulated accelerograms (see for instance Spanos and Loli (1985); Boore and Boomer (2005)). In this paper, the method proposed allows to directly take this correction into account in the construction of the pdf. Let $V(t)$ and $D(t)$ be the velocity and the displacement stochastic processes indexed by $[0, T]$. Assuming that $V(0)=D(0)=0$ almost surely, it can easily be proven that

$$
\begin{aligned}
& V(t)=\int_{0}^{t} A(\tau) d \tau, \\
& D(t)=\int_{0}^{t} V(\tau) d \tau,
\end{aligned}
$$

in which $\{A(t), t \in[0, T]\}$ is the acceleration stochastic process. Performing an integration by parts in the right-hand side of Eq. (32) and using Eq. (31) yield,

$$
D(t)=\int_{0}^{t}(t-\tau) A(\tau) d \tau .
$$

Using the time sampling $t_{j}=j \Delta t$ for $j=1, \ldots, N$ and the corresponding sampling $A_{j}=A\left(t_{j}\right)$, the following discretization of Eqs. (31) and (33) are then introduced,

$$
\begin{gathered}
I_{n}^{(1)}(\boldsymbol{A})=V\left(t_{n}\right) \simeq \Delta t \sum_{j=1}^{n} A_{j}, \\
I_{n}^{(2)}(\boldsymbol{A})=D\left(t_{n}\right) \simeq(\Delta t)^{2} \sum_{j=1}^{n}(n-j+1) A_{j},
\end{gathered}
$$

in which $\boldsymbol{A}=\left(A_{1}, \ldots, A_{N}\right)$. The zero end-velocity, $I_{N}^{(1)}(\boldsymbol{A})=V\left(t_{N}\right)$ and the zero end-displacement, $I_{N}^{(2)}(\boldsymbol{A})=D\left(t_{N}\right)$, are then specified in writing 
$I_{N}^{(1)}(\boldsymbol{A})=0$ and $I_{N}^{(2)}(\boldsymbol{A})=0$. These properties should be satisfied almost surely, which means that all the simulated trajectories of the acceleration stochastic process should satisfy this property. Three approaches could be used to take into account these almost sure properties through a constraint defined by Eq. (1). The first one would consist in performing a change of variable in order to impose the constraints related to the end-values, but the entropy defined in Eq. (4) is not invariant when operating a change of variable (see Kapur and Kevasan (1992)). The second one would consist in imposing this constraint in probability but would require to introduce a regularization. The third one, which is the one adopted in this paper, consists in imposing the constraint in the mean-square sense and not almost surely. Since random vector $\boldsymbol{A}$ is centered, then random variables $I_{N}^{(1)}(\boldsymbol{A})$ and $I_{N}^{(2)}(\boldsymbol{A})$ are also centered. We then introduce the following constraint,

$$
E\left\{\left(I_{N}^{(1)}(\boldsymbol{A})\right)^{2}\right\}=0 \quad, \quad E\left\{\left(I_{N}^{(2)}(\boldsymbol{A})\right)^{2}\right\}=0,
$$

which shows, using the Tchebychev inequality (DeGroot and Schervish (2011)), that $I_{N}^{(1)}(\boldsymbol{A})$ and $I_{N}^{(2)}(\boldsymbol{A})$ converge to 0 in probability.

\subsection{Mean value of the random VRS}

For $0<\omega_{\min }<\omega<\omega_{\max }$ and $0<\xi_{\min }<\xi<\xi_{\max }<1$, the random VRS $s(\omega, \xi ; A)$ of stochastic process $\{A(t), t \in[0, T]\}$ is defined by Clough and Penzien (1975)

$$
s(\omega, \xi ; A)=\omega \max _{t \in[0, T]}|y(t ; \omega, \xi, A)|,
$$

in which the stochastic process $\{y(t ; \omega, \xi, A), t \in[0, T]\}$ is defined by

$$
y(t ; \omega, \xi, A)=\int_{0}^{t} h(t-\tau ; \omega, \xi) A(\tau) d \tau,
$$

where

$$
h(t ; \omega, \xi)=-\mathbb{1}_{[0,+\infty[}(t) \frac{1}{\omega \sqrt{1-\xi^{2}}} \exp \{-\xi \omega t\} \sin \left\{\omega \sqrt{1-\xi^{2}} t\right\}
$$

in which the function $\mathbb{1}_{[0,+\infty[}(t)$ is equal to 1 if $t \in[0,+\infty[$ and is equal to 0 otherwise. In Eq. $(39), h(t ; \omega, \xi)$ is the impulse response function of a single degree of freedom oscillator with natural frequency $\omega$ and damping ratio $\xi$. Let $\kappa_{\omega}$ be the positive integer number of sampling points $\left\{\omega_{1}, \ldots, \omega_{\kappa_{\omega}}\right\}$ of the frequency (pulsation) interval $\left[\omega_{\min }, \omega_{\max }\right]$, such that $\omega_{\kappa_{\omega}}<\pi / \Delta t$. Similarly, let $\kappa_{\xi}$ be the positive integer number of sampling points $\left\{\xi_{1}, \ldots, \xi_{\kappa_{\xi}}\right\}$ of the damping rate interval $\left[\xi_{\min }, \xi_{\max }\right]$. Let be $\kappa=\kappa_{\omega} \times \kappa_{\xi}$. The discretization of Eqs. (37), (38) and (39) yields the random VRS vector $\boldsymbol{S}=\boldsymbol{s}(\boldsymbol{A})$ in which $s=\left(s_{1}, \ldots, s_{\kappa}\right)$ is a nonlinear mapping such that

$$
s_{j}(\boldsymbol{a})=s(\omega, \xi, \boldsymbol{a}) \text { for }(\omega, \xi)_{j} \text { in }\left\{\omega_{1}, \ldots, \omega_{\kappa_{\omega}}\right\} \times\left\{\xi_{1}, \ldots, \xi_{\kappa_{\xi}}\right\},
$$


in which

$$
s(\omega, \xi, \boldsymbol{a})=\omega \max \left\{\left|y_{1}(\omega, \xi, \boldsymbol{a})\right|, \ldots,\left|y_{N}(\omega, \xi, \boldsymbol{a})\right|\right\} \quad,
$$

with

$$
y_{i}(\omega, \xi, \boldsymbol{a})=\{[B(\omega, \xi)] \boldsymbol{a}\}_{i},
$$

and where $[B(\omega, \xi)]$ is a $(N \times N)$ real matrix defined by

$$
[B(\omega, \xi)]_{i j}=-\frac{\Delta t}{\omega \sqrt{1-\xi^{2}}} \exp \{-(i-j) \xi \omega \Delta t\} \sin \left\{(i-j) \omega \sqrt{1-\xi^{2}} \Delta t\right\}
$$

The available information related to the mean value of the random VRS is defined, for all $j$ in $\{1, \ldots, \kappa\}$, by

$$
E\left\{s_{j}(\boldsymbol{A})\right\}=\underline{s}_{j},
$$

where $\underline{s}=\left(\underline{s}_{1}, \ldots, \underline{s}_{\kappa}\right)$ is the mean VRS which is chosen as the target.

\subsection{Variability of the random VRS}

The constraint defined in Section 3.4 which concerns the mean value of the random VRS does not allow us to control the statistical fluctuations of the random VRS around its mean value. In this section, the variability of the random VRS is controlled by introducing a constraint related to the probability that the random VRS belongs to a region delimited by two given envelops. The VRS upper envelope is defined by the vector $\boldsymbol{s}^{\text {up }}=\left(s_{1}^{\text {up }}, \ldots, s_{\kappa}^{\text {up }}\right)$ and the VRS lower envelope is defined by the vector $s^{\text {low }}=\left(s_{1}^{\text {low }}, \ldots, s_{\kappa}^{\text {low }}\right)$. We then introduce the following constraint

$$
P\left(\left\{s_{1}^{\text {low }}<s_{1}(\boldsymbol{A})<s_{1}^{\text {up }}, \ldots, s_{\kappa}^{\text {low }}<s_{\kappa}(\boldsymbol{A})<s_{\kappa}^{\text {up }}\right\}\right)=p_{0},
$$

which can be rewritten as

$$
E\left\{\prod_{j=1}^{\kappa} \mathbb{1}_{\left[s_{j}^{\text {low }}, s_{j}^{\text {up }}\right]}\left(s_{j}(\boldsymbol{A})\right)\right\}=p_{0} .
$$

The indicator functions $x \mapsto \mathbb{1}_{\left[s_{j}^{\text {low }}, s_{j}^{\text {up }}\right]}(x)$ which are not differentiable are regularized by introducing the approximation $x \mapsto \mathbb{1}_{\left[s_{j}^{\text {low }}, s_{j}^{\text {up }}\right]}^{\text {reg }}(x)$ defined by

$$
\mathbb{1}_{\left[s_{j}^{\text {low }}, s_{j}^{\text {up }}\right]}^{\mathrm{reg}}(x)=\frac{1}{2}\left(\tanh \left(\frac{x-s_{j}^{\text {low }}}{\epsilon}\right)-\tanh \left(\frac{x-s_{j}^{\text {up }}}{\epsilon}\right)\right),
$$

in which $0<\epsilon \ll 1$ and for which the derivative with respect to $x$, denoted by $\delta^{\mathrm{reg}}(x)$, is such that

$$
\delta^{\mathrm{reg}}(x)=\frac{d}{d x} \mathbb{1}_{\left[s_{j}^{\mathrm{low}}, s_{j}^{\mathrm{up}}\right]}^{\mathrm{reg}}(x)=\frac{1}{2 \epsilon}\left(\tanh ^{2}\left(\frac{x-s_{j}^{\text {up }}}{\epsilon}\right)-\tanh ^{2}\left(\frac{x-s_{j}^{\text {low }}}{\epsilon}\right)\right) .
$$

The constraint defined by Eq. (46) is approximated by

$$
E\left\{\prod_{j=1}^{\kappa} \mathbb{1}_{\left[s_{j}^{\text {low }}, s_{j}^{\text {up }}\right]}^{\text {reg }}\left(s_{j}(\boldsymbol{A})\right)\right\}=p_{0} .
$$


3.6 Mean value of the random PGA and mean value of the random PGV

The PGA characterizes the maximum amplitude of the accelerogram. The random PGA, related to acceleration process $\{A(t), t \in[0, T]\}$, is defined by

$$
\operatorname{PGA}(A)=\max _{t \in[0, T]}|A(t)|
$$

In the regulation codes, this value is used to construct the target VRS. Nevertheless, even if the mean VRS of the simulated accelerograms matches perfectly the target VRS, the mean PGA of the simulated accelerograms does not match the PGA which has been used to construct the target VRS. In this section, we propose to enforce this matching. Using the time sampling of Eq. (50), the following constraint is introduced,

$$
E\left\{\max \left\{\left|A_{1}\right|, \ldots,\left|A_{N}\right|\right\}\right\}=\underline{\mathrm{PGA}},
$$

in which $\underline{\mathrm{PGA}}$ is the target value for the mean value of the PGA.

Concerning the random $\mathrm{PGV}$, which is defined by $\mathrm{PGV}(A)=\max \{|V(t)|, t \in$ $[0, T]\}$, its mean value is controlled by imposing the following constraint

$$
E\left\{\max \left\{\left|I_{1}^{(1)}(\boldsymbol{A})\right|, \ldots,\left|I_{N}^{(1)}(\boldsymbol{A})\right|\right\}\right\}=\underline{\mathrm{PGV}},
$$

in which $\underline{\mathrm{PGV}}$ is the target value for the mean value of the $\mathrm{PGV}$ and where $I_{j}^{(1)}(\boldsymbol{A})$ is defined by Eq. (34).

3.7 Mean value of the random CAV

The random CAV (EPRI (1991)) is defined as the integral of the absolute value of the random acceleration over time range $[0, T]$,

$$
\operatorname{CAV}(A)=\int_{0}^{T}|A(\tau)| d \tau
$$

The CAV is usually used for the risk assessment of nuclear power-plants. Using a discretization of Eq. (53), the corresponding constraint is introduced,

$$
E\left\{\Delta t \sum_{j=1}^{N}\left|A_{j}\right|\right\}=\underline{\mathrm{CAV}},
$$

in which $\underline{\mathrm{CAV}}$ is the target value for the mean value of the random $\mathrm{CAV}$. 
3.8 Mean value of the random AI

The random AI is defined (up to a multiplicative constant) as the integral of the square of the random acceleration over time range $[0, T]$,

$$
\mathrm{AI}(A)=\frac{\pi}{2 g} \int_{0}^{T} A^{2}(\tau) d \tau .
$$

where $g$ is the acceleration of gravity. The time discretization of Eq. (55) yields the following constraint,

$$
E\left\{\frac{\pi \Delta t}{2 g} \sum_{j=1}^{N} A_{j}^{2}\right\}=\underline{\mathrm{AI}},
$$

in which $\underline{\mathrm{AI}}$ is the target value for the mean value of the random AI.

\subsection{Remarks}

(1) It should be noted that the above list of constraints defined by the available information is not complete with respect to all the features of a natural accelerogram. As it will be seen for the application presented in Section 4, with such constraints, the generated accelerograms are not perfectly natural, but it is recalled that the main objective of the methodology proposed is to generate accelerograms which take into account the constraints imposed by regulation codes and/or by earthquake engineering. If necessary, the list of the constraints defined by the available information (given below) can be completed in order to take into account other features of a natural accelerogram such as a nonstationary phase, the zero-crossings of the acceleration, velocity and displacement, and so on.

(2) The constraint defined by Eq. (30) related to the mean AI is a linear combination of the constraint defined by Eq. (56) related to the variance of the components. As a consequence, if the constraints related to the variance of the components have already been taken into, the constraint related the AI is redundant and should not be taken into account in order to guaranty the uniqueness of the MaxEnt solution.

(3) All the information functions related to the constraints developed in Sections 3.2 to 3.8 are even functions in $\boldsymbol{a}$. Therefore, it can be shown that, if the available information concerns the mean value and additional constraints among those developed in Sections 3.2 to 3.8, then the Lagrange multipliers related to the mean value constraint are zero. In this case, the constraint related to the mean value can be omitted.

(4) Concerning the PGA, the PGV, the CAV and the AI, only constraints related to the mean value are developed in Sections 3.6 to 3.8. Additional constraints related to other statistics of these functions such as the variances and/or covariances could also be developed and be taken into account. 
(5) As it can be seen in Appendix A, most information functions related to the constraints developed above are nonquadratic functions yielding a nonGaussian pdf for random vector $\boldsymbol{A}$. The resulting pdf can be very complex and an adapted algorithm such as the one proposed in Section 2.2.3 is needed. It should also be noted that the classical methods based on a linear filtering of a Gaussian process are less time consuming than the proposed method but allows only the mean value of the VRS to be specified. The methodology proposed here allows a wide range of additional constraints to be taken into account in the construction of the pdf. These additional constraints such as the mean CAV can be very important for some particular industrial seismic designs (nuclear power plants for instance).

\section{Applications}

In this Section, several different sets of available information will be studied. The first case corresponds to constraints related to the mean value, the variance of the components and the zero end-velocity and the zero end-displacement. The other cases correspond to successive addition of available information. The time duration is $T=20 \mathrm{~s}$. The acceleration stochastic process is sampled at a time step, $\Delta t=0.0125 \mathrm{~s}$. We then have $N=1600$ (it is assumed that $A(0)=0 \mathrm{~ms}^{-2}$ almost surely).

4.1 Case 1. Constraints: mean value, variance of the components, zero end-velocity and zero end-displacement

This case yields a Gaussian centered random vector for which the mean VRS is not controlled. Therefore it is not suitable for a risk assessment. Nevertheless, since it corresponds to the Gaussian case, the Lagrange multipliers of the MaxEnt pdf can be computed very easily (see Section 2.2.2). These optimal Lagrange multipliers will then be used in the next sections devoted to nonGaussian MaxEnt pdf in order to construct the initial values of the Lagrange multipliers. The available information is then given by Eqs. (29), (30) and (36) for which the standard deviation $\left\{\sigma_{j}, j=1, \ldots, N\right\}$ is defined by $\sigma_{j}=$ $\lambda_{3}\left((j \Delta) t^{\lambda_{1}}\right) \exp \left(-\lambda_{1}(j \Delta t)\right)$ with $\lambda_{1}=2.05, \lambda_{2}=0.51$ and $\lambda_{3}=0.87$. This target standard deviation is plotted in Fig. 1. As explained in Section 2.2.2, since random vector $\boldsymbol{A}$ is centered, the Lagrange multiplier related to the mean vector is zero and therefore, the available information related to the mean vector is useless. Then, $\mu=N+2$ and the matrix $\left[\mathcal{K}_{\boldsymbol{\lambda}}\right]$ is defined by

$$
\left[\mathcal{K}_{\boldsymbol{\lambda}}\right]=\sum_{j=1}^{N} \lambda_{j}\left[K_{j}^{\mathrm{var}}\right]+\sum_{j=1}^{2} \lambda_{N+j}\left[K_{j}^{\mathrm{int}}\right] .
$$

The admissible space $\mathcal{L}_{\mu}$ of vector $\boldsymbol{\lambda}=\left(\lambda_{1}, \ldots, \lambda_{\mu}\right)$ is defined as $\left.\mathcal{L}_{\mu}=\right] 0,+\infty\left[{ }^{\mu}\right.$. It can easily be proven that (1) the $N+2$ matrices $\left\{\left[K_{j}^{\mathrm{var}}\right]\right\}_{j=1, \ldots, N},\left[K_{1}^{\text {int }}\right]$ and 


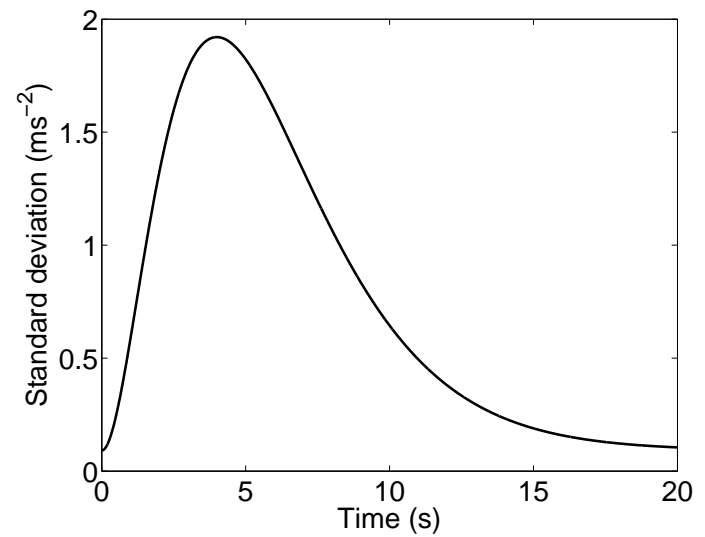

Fig. 1 Case 1: target standard deviation.

$\left[K_{2}^{\text {int }}\right]$ are algebraically independent and $(2)$ the matrix $\left[\mathcal{K}_{\boldsymbol{\lambda}}\right]$ is positive definite for all $\boldsymbol{\lambda}$ in $\mathcal{L}_{\mu}$. The methodology developed in Section 2.2 is applied using 30 iterations. Figure 2 shows two independent realizations of the random vector $\boldsymbol{A}_{\boldsymbol{\lambda}_{\text {sol }}}$ which is generated using a classical generator for Gaussian random vector and which are representative of two independent realizations of a random accelerogram. The corresponding trajectories of the velocity times series and of the displacement times series are plotted in Figs. 3 and 4. As expected, it can be seen that the end-velocity and the end-displacements are equal to zero.
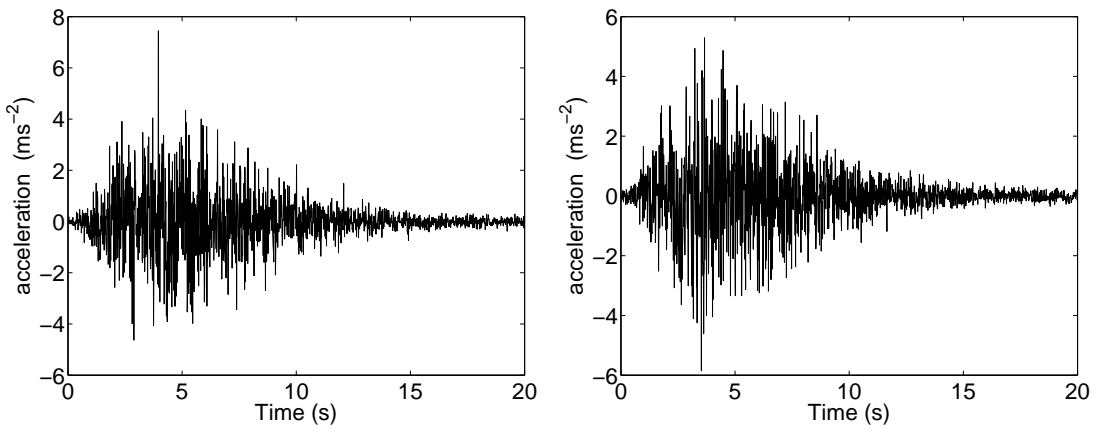

Fig. 2 Case 1: two independent realizations of the stochastic accelerogram. 

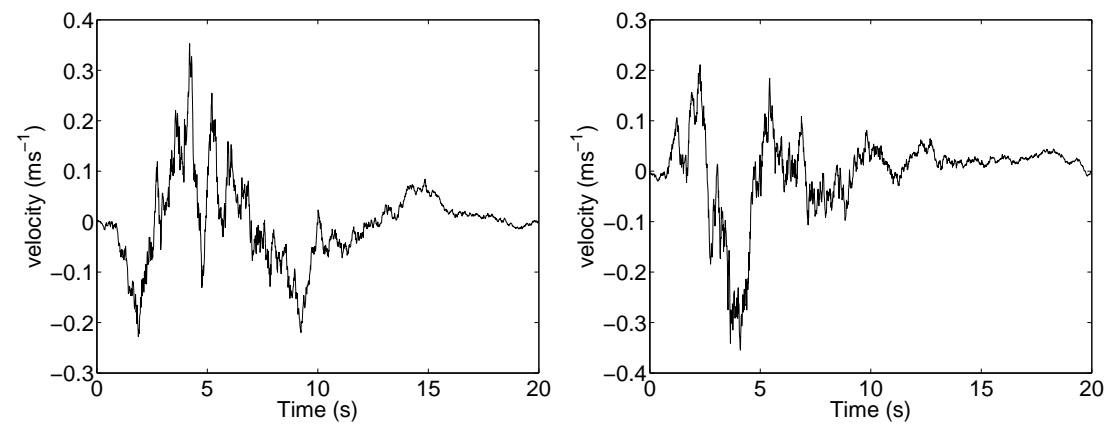

Fig. 3 Case 1: two independent realizations of the stochastic velocity.
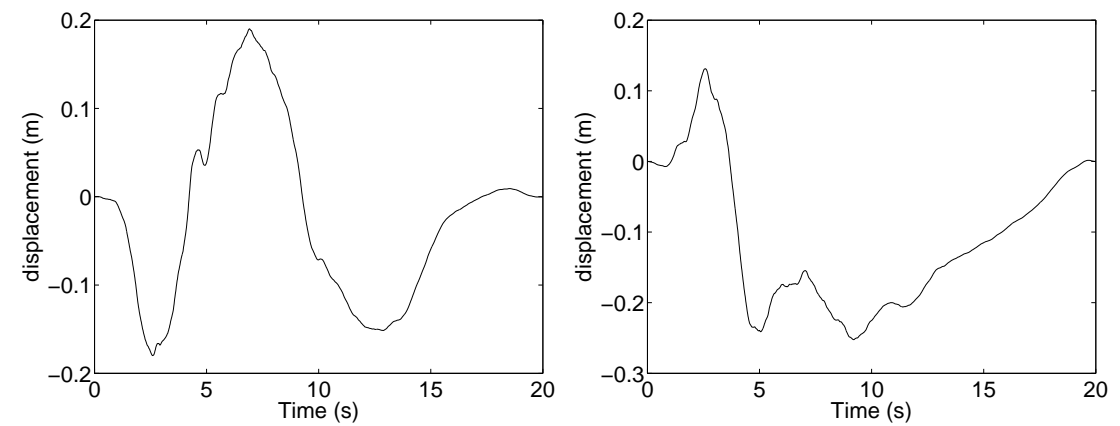

Fig. 4 Case 1: two independent realizations of the stochastic displacement.

\subsection{Case 2. Constraints: Case 1 and mean VRS}

The VRS is constructed following the Eurocode 8 for a A-type soil and a PGA equal to $5 \mathrm{~ms}^{-2}$. It is defined for 1 value of the damping ratio: 0.05 , and for 20 values of the angular frequency: 1.04, 1.34, 1.73, 2.23, 2.86, 3.69, $4.74,6.11,7.86,10.11,13.01,16.74,21.53,27.70,35.64,45.86,59.00,75.91$, 97.67 and $125.66($ in $\mathrm{rad} / \mathrm{s})$. We then have $\kappa=\kappa_{\omega} \times \kappa_{\xi}=20$. The target mean VRS, $\underline{s}=\left(\underline{s}_{1}, \ldots, \underline{s}_{\kappa}\right)$, is plotted in Fig. 5. Figure 6 displays the comparison between the target mean VRS of the present Case 2 and the mean value of the VRS simulated with Case 1 (for which no constraint related to the VRS is introduced). It can be seen that the two graphs are completely different and is due to the fact that the mean VRS is not taken into account in Case 1. For the identification of the Lagrange multipliers, the algorithm developed in Section 2.2.3 is used. For the initial value $\boldsymbol{\lambda}_{\text {init }}$ of the Lagrange multipliers, the nonlinear part $\boldsymbol{\lambda}_{\mathrm{NL} \text {,init }}$ is zero while the linear part $\boldsymbol{\lambda}_{\mathrm{L} \text {,init }}$ is the result of the identification carried out in Section 4.1. As explained in Section 2.2.3, $\boldsymbol{U}_{0, \text { init }} \sim$ $\mathcal{N}\left(\mathbf{0},\left[\mathcal{K}_{\boldsymbol{\lambda}_{\mathrm{L}, \mathrm{init}}}\right]^{-1}\right)$. At each iteration, the step size is $\Delta r_{\boldsymbol{\lambda}}=2 \pi /\left(\beta \sqrt{2 \lambda_{\max }}\right)$, in which $\lambda_{\text {max }}$ is the maximum of the components of vector $\lambda_{L}$ and $\beta=80$. At each iteration, the damping matrix $\left[D_{\boldsymbol{\lambda}}\right]$ is chosen as a diagonal matrix 


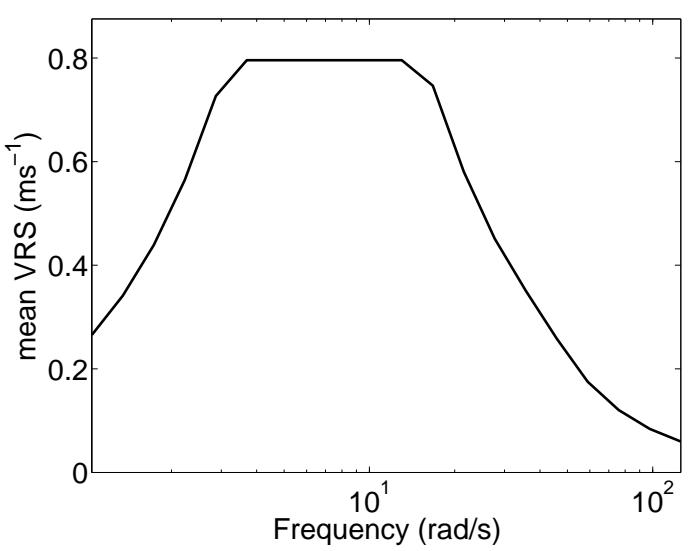

Fig. 5 Target mean VRS for Case 2.

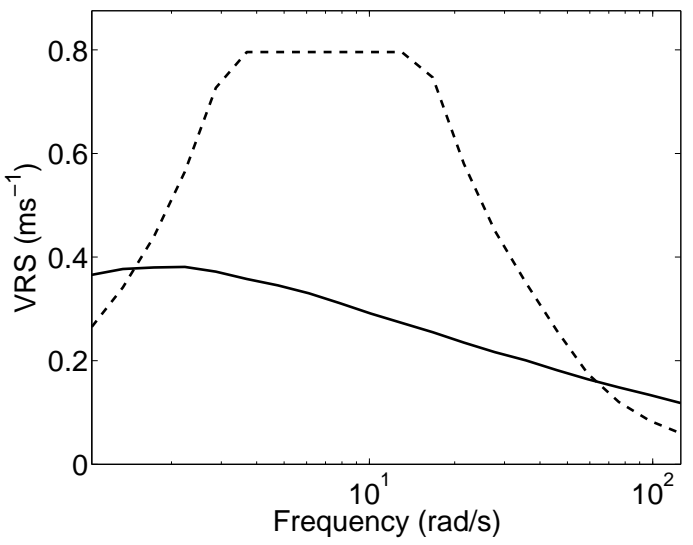

Fig. 6 Mean VRS: target (dashed line) of Case 2 and estimation given by Case 1 (solid line).

such that $\left[D_{\boldsymbol{\lambda}}\right]_{i i}=2 \xi_{\text {ito }} \sqrt{2\left\{\boldsymbol{\lambda}_{L}\right\}_{i}}$, in which $\xi_{\text {ito }}=0.7$. For the ISDE, the number of integration steps is $M=600$. At each iteration, $n_{s}=900$ Monte Carlo simulations are carried out. The under-relaxation parameter is fixed at $\alpha=0.3$. Figure 7 displays the comparison of the estimated standard deviation with the target values. Figure 8 displays the comparison of the mean VRS with the target mean VRS. Figures 7 and 8 shows a good matching for both the standard deviations and the mean VRS.

\subsection{Case 3. Constraints: Case 2 and variance of the VRS}

Let $\boldsymbol{s}^{\text {low }}$ be the lower envelop defined by $\boldsymbol{s}^{\text {low }}=0.5 \times \underline{s}$ and $\boldsymbol{s}^{\text {up }}$ be the upper envelop defined by $\boldsymbol{s}^{\mathrm{up}}=1.5 \times \underline{s}$. Figure 9 shows these two envelops and 100 


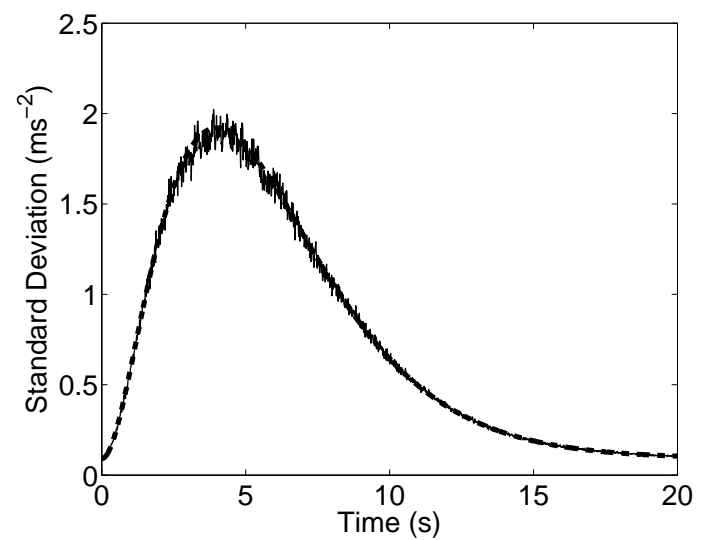

Fig. 7 Standard deviation for Case 2: target (thick dashed line) and estimation (thin solid line).

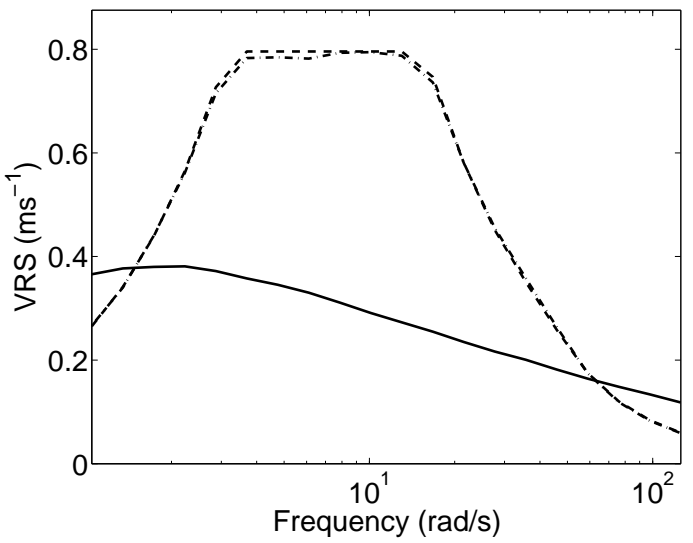

Fig. 8 Mean VRS: target (dashed line) for Case 2, estimation for Case 1 (solid line), estimation for Case 2 (mixed line).

trajectories of the random VRS constructed with the constraints of Case 2. In Fig. 9, it can be seen that even if a good matching of the mean VRS is obtained with the target VRS (see Section 4.2), then the statistical fluctuations around the mean VRS are large and several trajectories are out of the region delimited by the two envelops. For this case, the probability that the random VRS belongs to the region delimited by two envelops has been estimated using the Monte Carlo simulation method. The estimated value is $p_{0}=4.09 \times 10^{-2}$. In the present Case 3, the constraint defined by Eq. (49) is introduced with $p_{0}=$ $9.0 \times 10^{-2}$ and $\epsilon=0.07$. The parameters of the algorithm for the identification of the Lagrange multipliers are the same as those chosen in Section 4.2. Figure 10 displays 100 trajectories of the random VRS and the envelops $s^{\text {low }}$ and $s^{\text {up }}$. In comparison with Fig. 9, it can be seen that the statistical fluctuations 


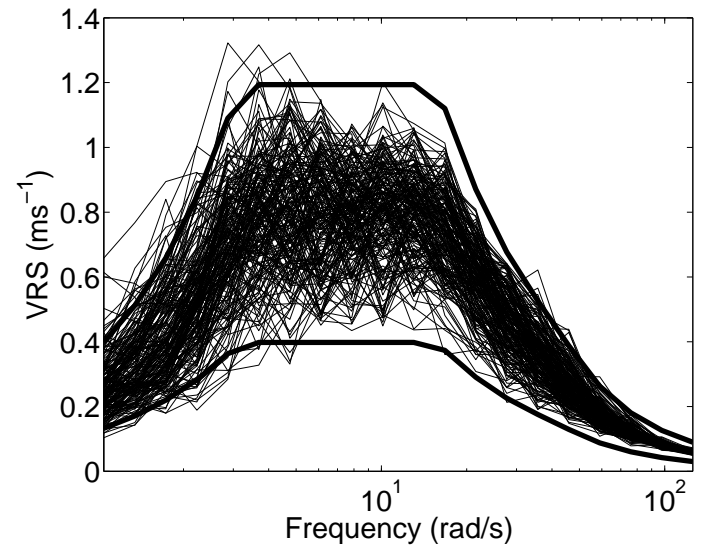

Fig. 9 Random VRS without constraint on the variance of the VRS (only constraints of Case 2): 100 trajectories (thin lines), lower and upper envelops (thick lines).

around the mean VRS has been reduced and all the trajectories are inside region delimited by the envelops. We have $E\left\{g_{\mathrm{NL}}^{\mathrm{VRS}-\mathrm{ENV}}(\boldsymbol{A})\right\}=8.99 \times 10^{-2}$

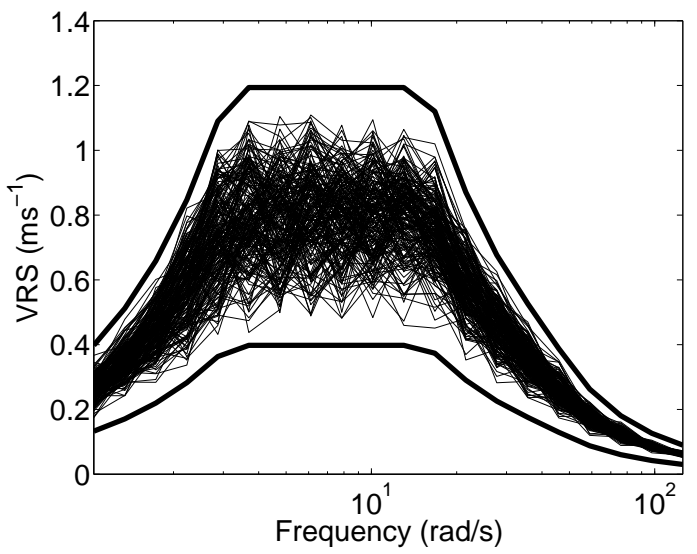

Fig. 10 Random VRS for Case 3: 100 trajectories (thin lines), lower and upper envelop (thick lines).

which is very close to the target value which is $9.0 \times 10^{-2}$.

4.4 Case 4. Constraints: Case 3 and mean PGA, mean PGV and mean CAV

In this section additional constraints to Case 3 are imposed in order to control the mean values for the PGA, PGV and CAV. For Case 3 presented in 
Section 4.3, the mean values of the PGA, PGV and CAV are estimated using Eqs. (51), (52) and (54) respectively, and we have $E\left\{g_{\mathrm{NL}}^{\mathrm{PGA}}(\boldsymbol{A})\right\}=6.30 \mathrm{~ms}^{-2}$, $E\left\{g_{\mathrm{NL}}^{\mathrm{PGV}}(\boldsymbol{A})\right\}=0.56 \mathrm{~ms}^{-1}$ and $E\left\{g_{\mathrm{NL}}^{\mathrm{CAV}}(\boldsymbol{A})\right\}=11.97 \mathrm{~ms}^{-1}$. It can be seen that the mean value of the PGA does not correspond to the value of the PGA that has been used to construct the target VRS in Section $4.2\left(5 \mathrm{~ms}^{-2}\right)$. In this section, this matching is enforced by imposing the constraint defined by Eq. (51) with $\underline{\mathrm{PGA}}=5 \mathrm{~ms}^{-2}$. The following values $\underline{\mathrm{PGV}}=0.45 \mathrm{~ms}^{-1}$ and $\underline{\mathrm{CAV}}=13 \mathrm{~ms}^{-2}$ are also imposed. For the identification of the Lagrange multipliers, the parameters of the algorithm are the same as those chosen in Section 4.2. Table 2 summarizes the results. It can be seen a good matching of the estimated means values of the PGA, PGV and CAV with the target values. Figure 11 shows two independent realizations of the random vector $\boldsymbol{A}_{\boldsymbol{\lambda}_{\text {sol }}}$ which are representative of two independent realizations of the stochastic accelerogram. The corresponding trajectories of the stochastic velocity and the stochastic displacement associated with the stochastic accelerogram are plotted in Figs. 12 and 13. As expected, it can be seen that the end-velocity and the end-displacement are equal to zero. We now verify that the additional constraints related to the the PGA, PGV and CAV do not corrupt the previous good matching for the variance of the components and for the mean VRS. Figure 14 displays a comparison of the estimated standard deviation of the components with the target values. Figure 15 compares the mean VRS with the target mean VRS. Figure 16 shows 100 trajectories of the random VRS and the envelops $\boldsymbol{s}^{\text {low }}$ and $\boldsymbol{s}^{\text {up }}$. The probability of being completely inside the envelops is $E\left\{g_{\mathrm{NL}}^{\mathrm{VRS}-\mathrm{ENV}}(\boldsymbol{A})\right\}=8.97 \times 10^{-2}$ (the target value is $\left.9.0 \times 10^{-2}\right)$. It can be seen in Figs. 14 to 16 that the results are still very good.

Table 2 Summary of Cases 1-4: mean PGA, PGV and CAV, and their target values.

\begin{tabular}{|l|c|c|c|c|c|}
\hline Constraint & Case 1 & Case 2 & Case 3 & Case 4 & Target \\
\hline$p_{0}$ & $1.25 \times 10^{-7}$ & 0.041 & 0.0899 & 0.0897 & 0.09 \\
\hline Mean PGA $\left(\mathrm{ms}^{-2}\right)$ & 5.68 & 6.12 & 6.30 & 4.98 & 5.00 \\
\hline Mean PGV $\left(\mathrm{ms}^{-1}\right)$ & 0.34 & 0.55 & 0.56 & 0.45 & 0.45 \\
\hline Mean CAV $\left(\mathrm{ms}^{-1}\right)$ & 12.06 & 11.87 & 11.97 & 13.04 & 13.0 \\
\hline
\end{tabular}

\subsection{Remark}

The results presented in Section 4.4 show a very good agreement between the target values and the values estimated using the generated accelerograms. Nevertheless, in Figs. 11-13, it can be seen that the generated trajectories are not perfectly natural. For instance, spurious low-frequency content appears at the beginning of the signal. Such low-frequency content should appear later in the signal. The time dependence of the velocity (or response) spectrum has been studied in the literature in which natural accelerograms have been analyzed 

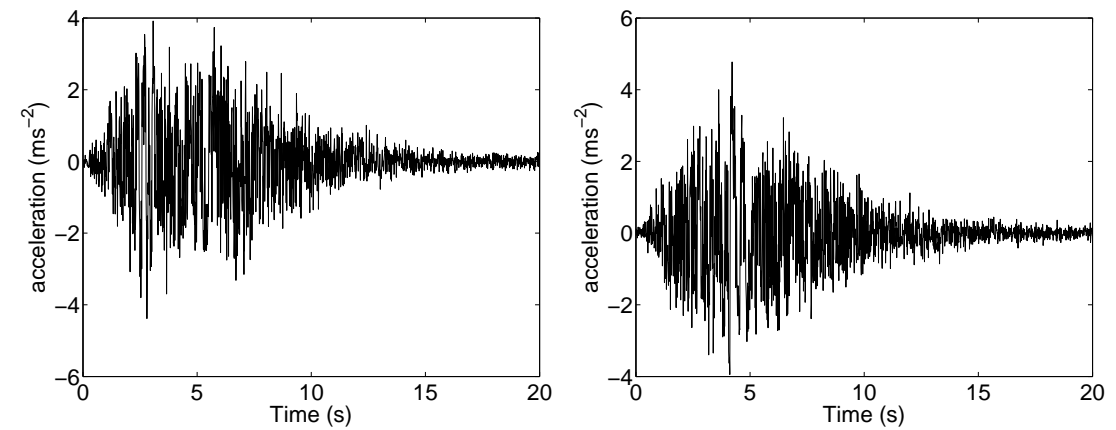

Fig. 11 Case 4: two independent realizations of the stochastic accelerogram.
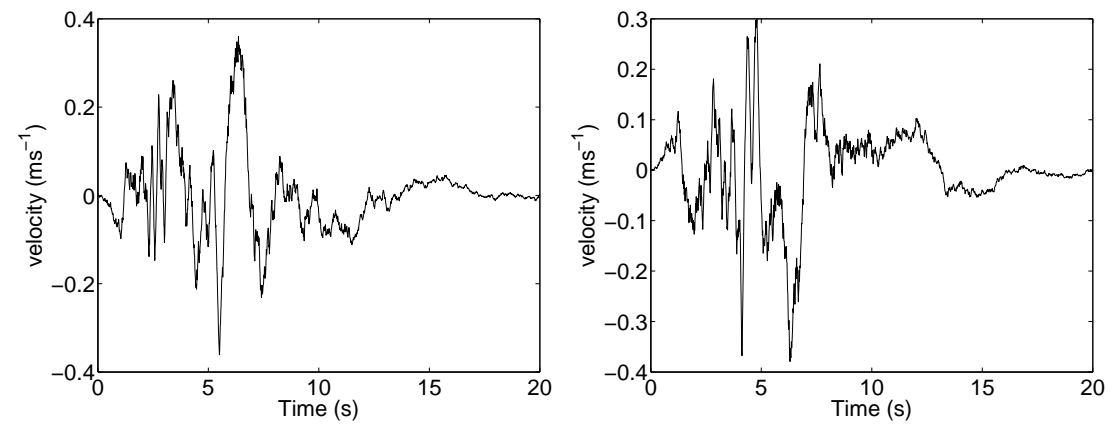

Fig. 12 Case 4: two independent realizations of the stochastic velocity.
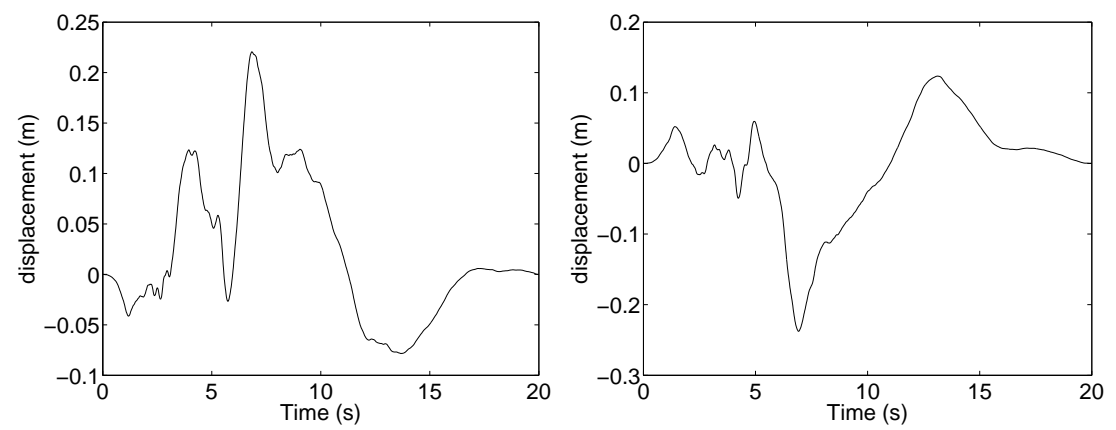

Fig. 13 Case 4: two independent realizations of the stochastic displacement.

in the time-frequency domain (see for instance, Spanos et al (2007)). With adapted constraints, it can be expected that the higher frequencies prevail during the "growth phase" of the accelerograms and then decay following a given rate. Such a rate could certainly be controlled but its analysis is beyond the scope of the present paper. In the application presented in this paper, the frequential content of the trajectories is controlled by the Eurocode VRS. It 


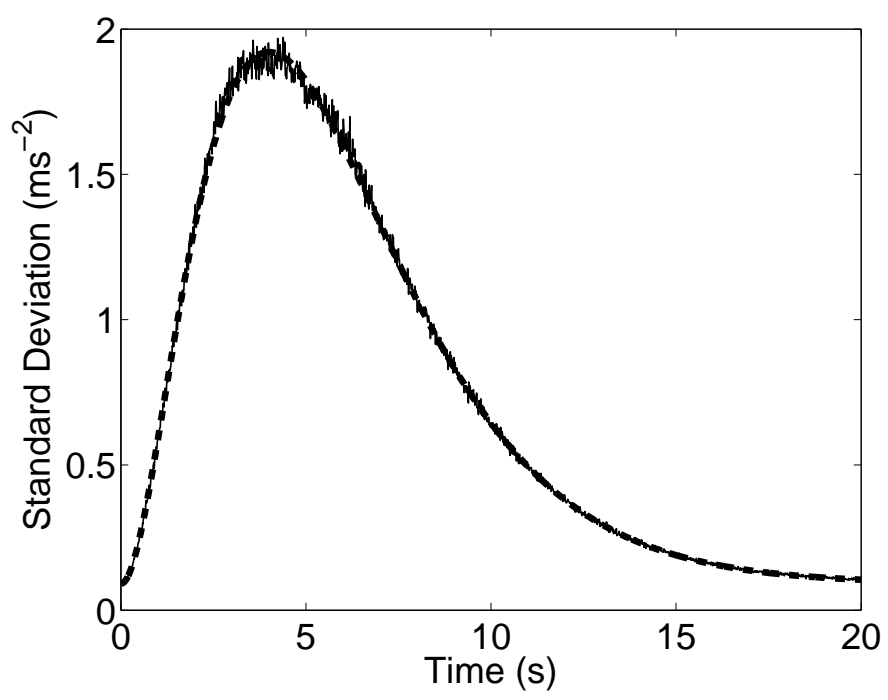

Fig. 14 Variance: Target (thick dashed line) and estimation (thin solid line).

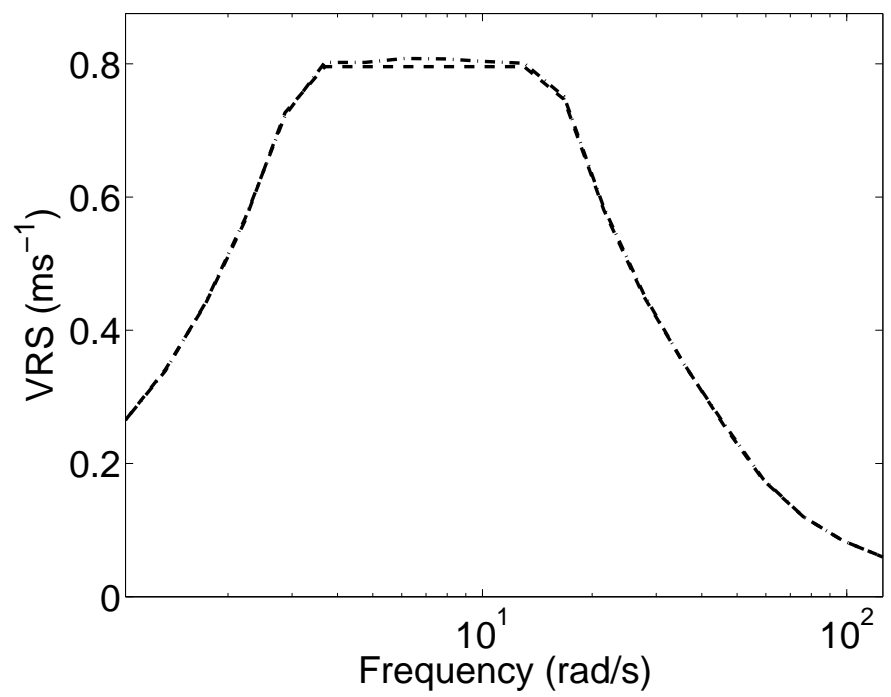

Fig. 15 Mean VRS: Target (dashed line), estimation (mixed line).

would thus seem that the Eurocode-VRS constraint is not sufficient to generate accelerograms with a natural frequency content. A time-dependent VRS could provide better trajectories. Such a constraint would consist in replacing Eq. (44) by a constraint of the following type,

$$
E\left\{s_{j}^{i}(\boldsymbol{A})\right\}=\underline{s}_{j}^{i},
$$




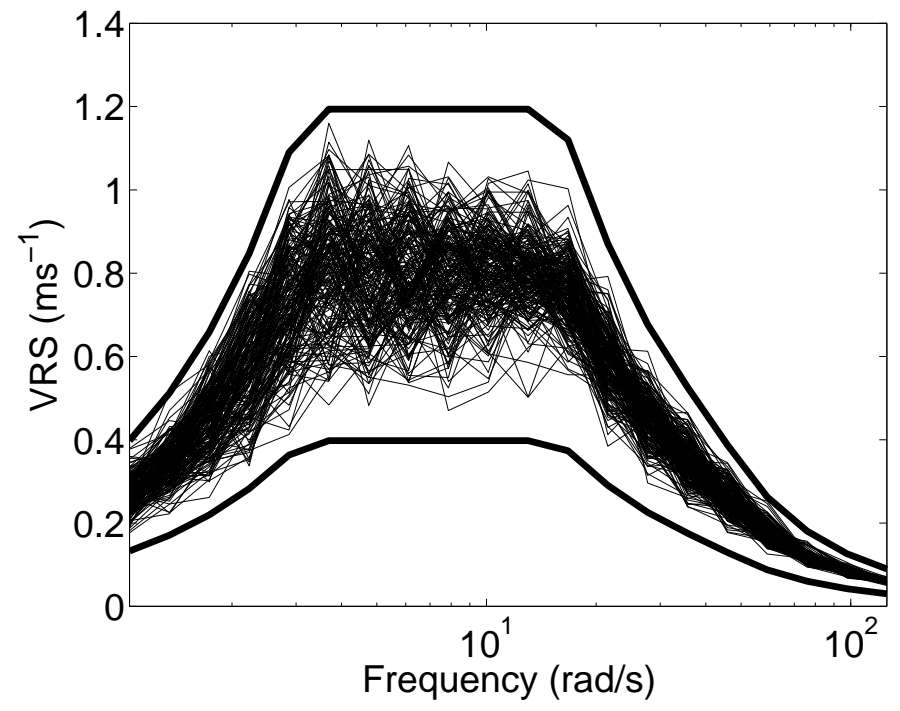

Fig. 16 Random VRS for Case 4: 100 trajectories (thin lines), lower and upper envelop (thick lines).

for all $i$ in $\{1, \ldots, N\}$ and for all $j$ in $\{1, \ldots, \kappa\}$, in which $s_{j}^{i}(\boldsymbol{A})$ would be the time sampling of the instantaneous VRS such that

$$
s_{j}^{i}(\boldsymbol{A})=s\left(t_{i}, \omega, \xi, \boldsymbol{A}\right) \quad \text { for }(\omega, \xi)_{j} \text { in }\left\{\omega_{1}, \ldots, \omega_{\kappa_{\omega}}\right\} \times\left\{\xi_{1}, \ldots, \xi_{\kappa_{\xi}}\right\},
$$

and where $\underline{s}_{j}^{i}$ would be the corresponding target. The introduction of an instantaneous VRS, $s(t, \omega, \xi, \boldsymbol{A})$, would allow the frequential content to be modified as a function of time $t$. The equation relating $\boldsymbol{A}$ to $s_{j}^{i}$ would have to be constructed using an adapted representation of stochastic process $\{A(t), t \in$ $[0, T]\}$, such as a wavelet representation (see for instance Giaralis and Spanos $(2009))$.

\section{Conclusions}

A new methodology has been presented for the generation of accelerograms compatible with a wide range of specifications. The proposed method allows the specifications to be directly taken into account in the probability distribution of the acceleration time series and therefore does not need to a posteriori corrections in order that the generated accelerograms match the specifications. The associated problem of the high stochastic dimension has been solved by introducing an adapted algorithm based on the resolution of an Itô stochastic differential equation. The method has the advantage of being very versatile and more constraints can easily be taken into account in addition to those introduced in this paper. The applications presented show a good accuracy 
of the method despite the presence of numerous nonlinear constraints. Therefore, from an engineering point of view for which the specification matching is of most importance, the proposed method can directly be used to perform a seismic design of a structure in nonlinear dynamics. From a physical point of view, the acceleration trajectories that have been generated could be improved to be more natural by introducing additional constraints related to the time dependence of the frequency content of the accelerograms.

\section{A Information functions and gradients}

In this appendix, for each constraint introduced in Section 3, the information functions $\boldsymbol{\nabla}_{\boldsymbol{a}} \Phi(\boldsymbol{a}, \boldsymbol{\lambda})$ are specified. Furthermore for $\boldsymbol{\lambda}$ fixed, $\left[K_{1}\right], \ldots,\left[K_{\mu_{\mathrm{L}}}\right]$ and $\boldsymbol{\nabla}_{\boldsymbol{a}}\left\{\boldsymbol{g}_{\mathrm{NL}}(\boldsymbol{a})\right\}_{j}$ for $j=1, \ldots, \mu_{\mathrm{NL}}$ are also specified.

\section{A.1 Mean value}

The information function corresponding to the mean value is

$$
\left\{\boldsymbol{g}_{\mathrm{NL}}^{\text {mean }}(\boldsymbol{a})\right\}_{j}=a_{j},
$$

yielding

$$
\left\{\nabla_{\boldsymbol{a}}\left\{\boldsymbol{g}_{\mathrm{NL}}^{\mathrm{mean}}(\boldsymbol{a})\right\}_{j}\right\}_{i}=\delta_{i j},
$$

in which $\delta_{i j}$ is the Kronecker symbol.

\section{A.2 Variance of the components}

The information function corresponding to the variance of the components of random vector $\boldsymbol{A}$ is

$$
\left\{\boldsymbol{g}_{\mathrm{L}}^{\operatorname{var}}(\boldsymbol{a})\right\}_{j}=a_{j}^{2} .
$$

Consequently, for all $i$ and $j$ in $\{1, \ldots, N\}$, we have

$$
\left\{\nabla_{\boldsymbol{a}}\left\{\boldsymbol{g}_{\mathrm{L}}^{\mathrm{var}}(\boldsymbol{a})\right\}_{j}\right\}_{i}=2 a_{j} \delta_{i j} \quad, \quad\left[K_{j}^{\mathrm{var}}\right]_{i k}=2 \delta_{k j} \delta_{i j} .
$$

A.3 Variance of the end-velocity and the end-displacement

The information function is written as

$$
\left\{\boldsymbol{g}_{\mathrm{L}}^{\text {int }}(\boldsymbol{a})\right\}_{1}=\left(I_{N}^{(1)}(\boldsymbol{a})\right)^{2} \quad, \quad\left\{\boldsymbol{g}_{\mathrm{L}}^{\text {int }}(\boldsymbol{a})\right\}_{2}=\left(I_{N}^{(2)}(\boldsymbol{a})\right)^{2},
$$

which are quadratic functions. Consequently, for all $i$ and $j$ in $\{1, \ldots, N\}$, we have

$$
\begin{gathered}
\left\{\nabla_{\boldsymbol{a}}\left\{\boldsymbol{g}_{\mathrm{L}}^{\mathrm{int}}(\boldsymbol{a})\right\}_{1}\right\}_{j}=2(\Delta t)^{2} \sum_{k=1}^{N} a_{k} . \\
\left\{\nabla_{\boldsymbol{a}}\left\{\boldsymbol{g}_{\mathrm{L}}^{\text {int }}(\boldsymbol{a})\right\}_{2}\right\}_{j}=2(\Delta t)^{4}(N-j+1) \sum_{k=1}^{N}(N-k+1) a_{k}, \\
{\left[K_{1}^{\text {int }}\right]_{j i}=2(\Delta t)^{2} \quad, \quad\left[K_{2}^{\text {int }}\right]_{j i}=2(\Delta t)^{4}(N-j+1)(N-i+1) .}
\end{gathered}
$$


A.4 Mean value of the random VRS

The information function related to the mean value of the random VRS is defined, for all $j$ in $\{1, \ldots, \kappa\}$, by

$$
\left\{\boldsymbol{g}_{\mathrm{NL}}^{\mathrm{VRS}}(\boldsymbol{a})\right\}_{j}=s_{j}(\boldsymbol{a}) .
$$

For fixed $j$ and for $(\omega \times \xi)=(\omega \times \xi)_{j}$, using Eq. (33), (34), (35) and the chain rules for the calculation of the gradient, it can be proven that

$$
\begin{aligned}
\left\{\boldsymbol{\nabla}_{\boldsymbol{a}}\left\{\boldsymbol{g}_{\mathrm{NLS}}^{\mathrm{VRS}}(\boldsymbol{a})\right\}_{j}\right\}_{i} & =\left\{\boldsymbol{\nabla}_{\boldsymbol{a}} s_{j}(\boldsymbol{a})\right\}_{i} \\
& =\omega[B(\omega, \xi)]_{q i} \operatorname{sgn}\left\{y_{q}(\omega, \xi, \boldsymbol{a})\right\},
\end{aligned}
$$

in which $\operatorname{sgn}\left\{y_{q}(\omega, \xi, \boldsymbol{a})\right\}$ is the sign of $y_{q}(\omega, \xi, \boldsymbol{a})$ defined in Eq. (42), and where

$$
q=\operatorname{imax}\left\{\left|y_{1}(\omega, \xi, \boldsymbol{a})\right|, \ldots,\left|y_{N}(\omega, \xi, \boldsymbol{a})\right|\right\},
$$

where imax is a function with values in $\{1, \ldots, N\}$ such that

$$
\operatorname{imax}\left(x_{1}, \ldots, x_{N}\right)=\operatorname{imax}(\boldsymbol{x})=\arg \max _{i \in\{1, \ldots, N\}} x_{i} .
$$

For $\|\boldsymbol{a}\| \neq 0$, it should be noted that the derivative given by Eq. (69) holds if $q$ constructed using Eqs. (70) and (71) is unique. This derivative is used for $\boldsymbol{a}$ equal to $\boldsymbol{U}^{k, \ell}$ in which $\boldsymbol{U}^{k, \ell}$ is a given realization $\left\{\boldsymbol{U}^{k, \ell}, k=1, \ldots, M-1\right\}$ of the time series $\left\{\boldsymbol{U}^{k}, k=1, \ldots, M-1\right\}$. For a given realization $\ell$, the probability that there exist two solutions for Eq. (71) is zero. For $\|\boldsymbol{a}\|=0$, this derivative does not exist but, since the initial condition is such that $\|\boldsymbol{a}\| \neq 0$, the probability that $\left\|\boldsymbol{U}^{k, \ell}\right\|$ be equal to zero is zero.

\section{A.5 Variability of the random VRS}

The information function corresponding to the variability of the random VRS is defined by

$$
g_{\mathrm{NL}}^{\mathrm{VRS}-\mathrm{ENV}}(\boldsymbol{a})=\prod_{j=1}^{\kappa} \mathbb{1}_{\left[s_{j}^{\mathrm{low}}, s_{j}^{\mathrm{up}}\right]}^{\mathrm{reg}}\left(s_{j}(\boldsymbol{a})\right)
$$

Its gradient function is written as

$$
\left\{\boldsymbol{\nabla}_{\boldsymbol{a}} g_{\mathrm{NL}}^{\mathrm{VRS}-\mathrm{ENV}}(\boldsymbol{a})\right\}_{i}=g_{\mathrm{NL}}^{\mathrm{VRS}-\mathrm{ENV}}(\boldsymbol{a}) \sum_{j=1}^{\kappa}\left\{\boldsymbol{\nabla}_{\boldsymbol{a}} s_{j}(\boldsymbol{a})\right\}_{i} \frac{\delta^{\mathrm{reg}}\left(s_{j}(\boldsymbol{a})\right)}{\mathbb{1}_{\left[s_{j}^{\mathrm{reg}}, s_{j}^{\text {up }}\right]}^{\mathrm{rg}}\left(s_{j}(\boldsymbol{a})\right)},
$$

in which $\left\{\nabla_{\boldsymbol{a}} s_{j}(\boldsymbol{a})\right\}_{i}$ is defined by Eq. (69).

A.6 Mean value of the random PGA and mean value of the random PGV

The information function corresponding to the mean value of the random PGA is

$$
g_{\mathrm{NL}}^{\mathrm{PGA}}(\boldsymbol{a})=\max \left\{\left|a_{1}\right|, \ldots,\left|a_{N}\right|\right\} .
$$

and the gradient function is written as

$$
\left\{\nabla_{\boldsymbol{a}} g_{\mathrm{NL}}^{\mathrm{PGA}}(\boldsymbol{a})\right\}_{i}=\delta_{i q} \operatorname{sgn}\left\{a_{q}\right\}
$$


where $q=\operatorname{imax}\{\boldsymbol{a}\}$, in which the function $\boldsymbol{x} \mapsto \operatorname{imax}(\boldsymbol{x})$ is defined in Eq. (71). Concerning the random $\mathrm{PGV}$, the corresponding information function related to the mean value is defined by

$$
g_{\mathrm{NL}}^{\mathrm{PGV}}(\boldsymbol{a})=\max \left\{\left|I_{1}^{(1)}(\boldsymbol{a})\right|, \ldots,\left|I_{N}^{(1)}(\boldsymbol{a})\right|\right\},
$$

whose gradient function is written as

$$
\left\{\boldsymbol{\nabla}_{\boldsymbol{a}} g_{\mathrm{NL}}^{\mathrm{PGV}}(\boldsymbol{a})\right\}_{i}=\operatorname{sgn}\left\{I_{q}^{(1)}(\boldsymbol{a})\right\} \mathbb{1}_{[1, q]}(i),
$$

in which $q=\operatorname{imax}\left\{\left|I_{1}^{(1)}(\boldsymbol{a})\right|, \ldots,\left|I_{N}^{(1)}(\boldsymbol{a})\right|\right\}$.

\section{A.7 Mean value of the random CAV}

The corresponding information function related to the mean CAV is defined by

$$
g_{\mathrm{NL}}^{\mathrm{CAV}}(\boldsymbol{a})=\Delta t \sum_{j=1}^{N}\left|a_{j}\right|,
$$

and its gradient function is such that

$$
\left\{\boldsymbol{\nabla}_{\boldsymbol{a}} \boldsymbol{g}_{\mathrm{NL}}^{\mathrm{CAV}}(\boldsymbol{a})\right\}_{i}=\Delta t \operatorname{sgn}\left\{a_{i}\right\} .
$$

A.8 Mean value of the random AI

The information function related to the mean AI is defined by

$$
g_{\mathrm{L}}^{\mathrm{AI}}(\boldsymbol{a})=\frac{\pi \Delta t}{2 g} \sum_{j=1}^{N} a_{j}^{2},
$$

which is a quadratic function. For all $i$ and $j$ in $\{1, \ldots, N\}$, we have

$$
\left\{\nabla_{\boldsymbol{a}} g_{\mathrm{L}}^{\mathrm{AI}}(\boldsymbol{a})\right\}_{i}=\frac{\pi \Delta t}{g} a_{i} \quad, \quad\left[K^{\mathrm{AI}}\right]_{i j}=\frac{\pi \Delta t}{g} \delta_{i j} .
$$

\section{Acknowledgements}

This research was supported by the "Agence Nationale de la Recherche", Contract TYCHE, ANR-2010-BLAN-0904.

\section{References}

Agmon N, Alhassid Y, Levine RD (1979) An algorithm for finding the distribution of maximal entropy. Journal of Computational Physics 30:250-258

Ahmadi G (1979) Generation of artificial time-histories compatible with given response spectra - a review. Solid Mechanics Archives 4(3):207-239 
Boore DM, Boomer JJ (2005) Processing of strong motion accelerograms: needs, options and consequences. Soil Dynamics and Earthquake Engineering 25:93-115

Cacciola P (2010) A stochastic approach for generating spectrum compatible fully nonstationary earthquakes. Computers \& Structures 88(15-16):889-901

Cacciola P, Zentner I (2012) Generation of response-spectrum-compatible artificial earthquake accelerograms with random joint time-frequency distributions. Probabilistic Engineering Mechanics 28:52-58

CEN (2003) Eurocode 8: Design of Structures for Earthquake ResistancePart1: General Rules, Seismic Actions and Rules for Buildings. EN 1998-1: 2003 European Committee for Standardization. Brussels

Clough RW, Penzien J (1975) Dynamics of Structures. McGraw-Hill

DeGroot MH, Schervish MJ (2011) Probability and Statistics, 4th edn. Pearson

EPRI (1991) Standardization of the Cumulative Absolute Velocity, Report No. EPRI TR-100082-T2. Palo Alto, California

Giaralis A, Spanos PD (2009) Wavelet-based response spectrum compatible synthesis of accelerograms-eurocode application (ec8). Soil Dynamics and Earthquake Engineering 29(1):219-235

Giaralis A, Spanos PD (2012) Derivation of response spectrum compatible non-stationary stochastic processes relying on monte carlo-based peak factor estimation. Earthquake and Structures 3(3-4):581-609

Golan A, Judge G, Miller D (1996) Maximum entropy econometrics: robust estimation with limited data. Wiley, New York

Gu P, Wen YK (2007) A record-based method for the generation of tridirectional uniform hazard-response spectra and ground motions using the hilbert-huang transform. Bulletin of the Seismological Society of America 97(5):1539-1556

Hancock J, Watson-Lamprey J, Abrahamson NA, Bommer JJ, A Markartis EM, et al (2006) An improve method of matching response spectra of recorded earthquake ground motion using wavelets. Journal of Earthquake Engineering 10:67-89

Hastings WK (1970) Monte carlo sampling methods using markov chains and their applications. Biometrica 109:57-97

Iyengar RN, Rao PN (1979) Generation of spectrum compatible accelerograms. Journal of Earthquake Engineering \& Structural Dynamics 7(3):253-263

Jaynes ET (1954) Information theory and statistical mechanics. Physical Review 106(4):620-630

Kapur JN, Kevasan HK (1992) Entropy Optimization Principles with Applications. Academic Press, San Diego

Katsanos EI, Sextos AG, Manolis GD (2010) Selection of earthquake ground motion records: A state-of-the-art review from a structural engineering perspective. Soil Dynamics and Earthquake Engineering 10:157-169

Lin CCJ, Ghaboussi J (2001) Generating multiple spectrum compatible accelerograms using stochastic neural networks. Journal of Earthquake Engineering \& Structural Dynamics 30(7):1021-1042 
Mukherjee S, Gupta VK (2002) Wavelet-based generation of spectrumcompatible time-histories. Soil Dynamics and Earthquake Engineering 22:799-804

Naeim F, Alimoradi A, Pezeshk S (2004) Selection and scaling of ground motion time histories for structural design using genetic algorithms. Earthquake Spectra 20:413-426

Preumont A (1980) A method for the generation of artificial earthquake accelerograms. Nuclear Engineering and Design 59:357-368

Preumont A (1985) The generation of non-separable artificial earthquake accelerograms for the design of nuclear power plants. Nuclear Engineering and Design 88:59-67

Razaeian S, Der Kiureghian A (2008) A stochastic ground motion model with separable temporal and spectral nonstationarities. Journal of Earthquake Engineering \& Structural Dynamics 37(13):1565-1584

Rubinstein RY, Kroese DP (2008) Simulation and the Monte Carlo Method, 2nd edn. John Wiley and Sons, New York

Sabetta F, Pugliese A (1996) Estimation of response spectra and simulation of nonstationary earthquake groung motions. Bulletin of the Seismological Society of America 86:337-352

Shannon CE (1948) A mathematical theory of communication. Bell System Technology Journal 27:379-423 and 623-659

Shrinkhande M, Gupta VK (1996) On generating ensemble of design spectrumcompatible accelerograms. European Earthquake Engineering X(3):49-56

Soize C (2008) Construction of probability distributions in high dimension using the maximum entropy principle. applications to stochastic processes, random fields and random matrices. International Journal for Numerical Methods in Engineering 76(10):1583-1611

Soize C (2010) Information theory for generation of accelerograms associated with shock response spectra. Computer-Aided Civil and Infrastructure Engineering 25:334-347

Spanos PD, Loli LMV (1985) A statistical approach to generation of design spectrum compatible earthquake time histories. International Journal of Soil Dynamics and Earthquake Engineering 4(1):2-8

Spanos PD, Giaralis A, Politis NP (2007) Time-frequency representation of earthquake accelerograms and inelastic structural response records using the adaptive chirplet decomposition and empirical mode decomposition. Soil Dynamics and Earthquake Engineering 27:675-689

Zentner I, Poirion F (2012) Enrichment of seismic ground motion databases using karhunen-loève expansion. Journal of Earthquake Engineering \& Structural Dynamics 41(14):1945-1957 